\title{
The relationship between social support and mental health problems during pregnancy: a systematic review and meta-analysis
}

Asres Bedaso ${ }^{1,2^{*} \mathbb{D}}$, Jon Adams², Wenbo Peng ${ }^{2}$ and David Sibbritt ${ }^{2}$

\begin{abstract}
Background: Pregnancy is a time of profound physical and emotional change as well as an increased risk of mental illness. While strengthening social support is a common recommendation to reduce such mental health risk, no systematic review or meta-analysis has yet examined the relationship between social support and mental problems during pregnancy.

Methods: The PRISMA checklist was used as a guide to systematically review relevant peer-reviewed literature reporting primary data analyses. PubMed, Psych Info, MIDIRS, SCOPUS, and CINAHL database searches were conducted to retrieve research articles published between the years 2000 to 2019. The Newcastle-Ottawa Scale tool was used for quality appraisal and the meta-analysis was conducted using STATA. The $Q$ and the $I^{2}$ statistics were used to evaluate heterogeneity. A random-effects model was used to pool estimates. Publication bias was assessed using a funnel plot and Egger's regression test and adjusted using trim and Fill analysis.
\end{abstract}

Result: From the identified 3760 articles, 67 articles with 64,449 pregnant women were part of the current systematic review and meta-analysis. From the total 67 articles, 22 and 45 articles included in the narrative analysis and metaanalysis, respectively. From the total articles included in the narrative analysis, 20 articles reported a significant relationship between low social support and the risk of developing mental health problems (i.e. depression, anxiety, and self-harm) during pregnancy. After adjusting for publication bias, based on the results of the random-effect model, the pooled odds ratio (POR) of low social support was AOR: 1.18 (95\% Cl: 1.01, 1.41) for studies examining the relationship between low social support and antenatal depression and AOR: $1.97(95 \% \mathrm{Cl}: 1.34,2.92)$ for studies examining the relationship between low social support and antenatal anxiety.

Conclusion: Low social support shows significant associations with the risk of depression, anxiety, and self-harm during pregnancy. Policy-makers and those working on maternity care should consider the development of targeted social support programs with a view to helping reduce mental health problems amongst pregnant women.

\section{Plain language summary}

Pregnancy is a significant event for reproductive-age women. It is supplemented by hormonal changes and can represent a time of increased risk for the occurrence of mental illness like depression, anxiety and self-harm. Providing good social support for the pregnant mother reduce this risk and prevent pregnancy complication and adverse birth

*Correspondence: asresbedaso@gmail.com

${ }^{1}$ College of Medicine and Health Sciences, School of Nursing, Hawassa

University, Hawassa, Ethiopia

Full list of author information is available at the end of the article permits use, sharing, adaptation, distribution and reproduction in any medium or format, as long as you give appropriate credit to the original author(s) and the source, provide a link to the Creative Commons licence, and indicate if changes were made. The images or other third party material in this article are included in the article's Creative Commons licence, unless indicated otherwise in a credit line to the material. If material is not included in the article's Creative Commons licence and your intended use is not permitted by statutory regulation or exceeds the permitted use, you will need to obtain permission directly from the copyright holder. To view a copy of this licence, visit http://creativecommons.org/licenses/by/4.0/. The Creative Commons Public Domain Dedication waiver (http://creativeco mmons.org/publicdomain/zero/1.0/) applies to the data made available in this article, unless otherwise stated in a credit line to the data. 
outcome. However, no systematic review and/or meta-analysis has explored the associations between social support and mental illness (depression, anxiety, self-harm) among pregnant women. Therefore, this systematic review and meta-analysis aimed to examine the association between social support and mental illness (anxiety, depression, and self-harm) during pregnancy.

The review identified 67 relevant articles with 64,449 pregnant women, from PubMed, Psych Info, MIDIRS, SCOPUS, and CINAHL database. Of the total 67 articles, 22 articles included in the narrative review and 45 articles included in the meta-analysis. Among studies included in the narrative synthesis, a majority of them reported significant positive associations between low social support and antenatal depression, antenatal anxiety and self-harm during pregnancy. Further, the pooled estimates of the meta-analysis show that low social support had a significant positive association with antenatal depression (AOR: 1.18 (95\% Cl: 1.01, 1.41)) and antenatal anxiety (AOR: 1.97 (95\% Cl: 1.34, 2.92)). Therefore, maternal health professionals need to have discussions with pregnant women regarding the level and source of social support they receive. Maternal health professionals may also need to consider encouraging the social network of pregnant women to improve social support being given. Policy-makers and those working on maternity care should consider the development of targeted social support programs with a view to helping reduce mental health problems amongst pregnant women.

Keywords: Social support, Pregnancy, Mental illness, Anxiety, Depression, Self-harm, Systematic review, Meta-analysis

\section{Background}

Pregnant women are at increased risk of developing mental health problems such as depression, anxiety, and self-harm [1, 2]; a risk that can be exacerbated by different factors like financial and relationship issues and low social class [3-5]. The common mental health problems women experiencing during pregnancy are anxiety [6], depression [7] and self-harm [8]. Antenatal anxiety is defined as excess worries, concerns, and fears about pregnancy, childbirth, the health of the infant, and future parenting roles [9]. Individual studies have reported that the prevalence of antenatal anxiety range between 14 and $59 \%$ [10-13], while, a meta-analysis conducted on estimating the global prevalence of antenatal anxiety found that pooled prevalence of antenatal anxiety symptoms across all trimesters was $34.4 \%$ in low to middle-income countries and $19.4 \%$ in high-income countries [14].

Depression is the most prevalent mental health problem during pregnancy [15], characterized by symptoms such as depressed mood, low self-esteem, loss of interest, feelings of worthlessness, irritable mood, loss of appetite, feelings of fatigue, and poor concentration [16]. An umbrella review conducted on examining the global prevalence of antenatal depression reported $15-65 \%$, and $17 \%$ pooled prevalence of antenatal depression in low to middle-income countries and high-income countries respectively based on ten identified systematic reviews [17].

Self-harm during pregnancy is one of the indirect causes of maternal death, especially among those who already developed mental health problems. For example in a study conducted in Bangladesh, among depressed pregnant women, nearly $14 \%$ admitted due to thoughts of self-harm [18] and in high-income countries, suicidal ideation is experienced by 3 to $33 \%$ of pregnant women $[19,20]$. A global level review found that the prevalence of suicidal ideation during pregnancy and postpartum ranges from 5 to $14 \%$ [21].

Antenatal depression and anxiety negatively affect several obstetric and fetal outcomes and, if not effectively managed, can lead to pregnancy complications, postnatal mental health problems [22-27], and risk of impaired interaction between mother and infant [15, 28-30]. Mental illness during pregnancy is also associated with increased risk-taking behaviours such as smoking and the use of other substances that can thereby result in a poor quality of life of the mother $[6,31,32]$.

One common strategy to help prevent or reduce pregnancy complications and adverse birth outcomes as a consequence of mental illness is to provide strong social support for the pregnant mother $[6,33,34]$. Social support is characterized by the degree to which social relationships fill specific needs (e.g. emotional, instrumental, affectionate, and/or tangible social support) or the degree of social integration [35, 36]. Social support is assumed to improve individuals' positive interactions that can help reduce depression, stress, and anxiety, and therefore reduce the risk of adverse pregnancy and birth outcomes [37]. Social support can also provide an additional suitable coping mechanism for pregnant women to handle stressful events $[6,37]$.

Different epidemiological studies have revealed that low social support is significantly associated with depression [38-40] anxiety [41, 42] and self-harm [43] during pregnancy. However, no systematic review and/or meta-analysis has been conducted to collate and critically review findings from individual studies; making the available evidence more accessible to decision-makers 
and providing an estimate of the magnitude of the associations between social support and mental health problems like depression, anxiety, and/or self-harm among pregnant women. In direct response to this significant research gap, this systematic review and meta-analysis aimed at examining whether low social support is associated with an increased risk of mental health problems during pregnancy. We hypothesized that low social support is significantly associated with depression, anxiety and/or self-harm during pregnancy.

\section{Methods \\ Information source, search strategy and study selection process}

This systematic review and meta-analysis was conducted and results were reported following the PRISMA (Preferred Reporting Items for Systematic Reviews and Meta-Analyses) checklist [44] (Additional file 1). This systematic review and meta-analysis protocol has been registered in PROSPERO (CRD42020155981). All peerreviewed published articles were systematically searched through a number of electronic databases including PubMed, Maternal and Infant care database (MIDIRS), PsychINFO, SCOPUS, and CINAHL.

We used the following search terms and key words used for searching from the PubMed database: ((()((()("Depression" [Mesh] OR "Depressive Disorder" [Mesh] OR "Depressive Disorder, Major" [Mesh])) OR depression [Title/Abstract]) OR depressive symptom [Title/Abstract])) OR ((Anxiety disorder [Title/Abstract]) OR ((anxiety [Title/Abstract]) OR ("Anxiety" [Mesh] OR "Anxiety Disorders" [Mesh] $))))$ OR ((((((((("Self-Injurious Behavior" [Mesh]) OR self-harm [Title/Abstract]) OR ("Self-Mutilation" [Mesh])) OR suicide [Title/Abstract]) OR "Suicide" [Mesh])) OR "Mental Health" [Mesh])) AND ((()("Social Support" [Mesh] OR "Psychosocial Support Systems" [Mesh])) OR social support [Title/ Abstract]) OR Psychosocial support [Title/Abstract]) OR emotional support [Title/Abstract]) OR instrumental support [Title/Abstract])) AND (((("Pregnancy" [Mesh]) OR "Pregnant Women" [Mesh])) OR pregnancy [Title/ Abstract]) OR pregnant women [Title/Abstract]). For the other four electronic databases (CINAHL, MIDIRS, Psych INFO, and SCOPUS) specific database subject headings linked with the above terms and keywords were used. Search limits used in the databases include English literature and the period starting from January 1, 2000 to November 8, 2019. Also, we have manually searched the reference lists of included studies to identify additional articles. Using Covidence software [45], the identified publications were evaluated by their titles, abstract, duplication and full-text contents against the pre-specified inclusion and exclusion criteria.

We employed the PICO model to determine the eligibility for the study: population: (1) adults pregnant women aged $\geq 18$ years; (2) intervention(s)/exposure(s) group: pregnant women who receive low social support; (3) comparison group(s): pregnant women who receive high/good social support; (4) outcomes: depression/ depressive symptoms, anxiety disorder/anxiety symptoms and self-harm among pregnant women. The initial search and selection of studies were undertaken by $A B$. Full-text articles were later checked for their eligibility by two investigators (AB and WP). Disagreements were resolved through discussion with a third and fourth investigator (JA, DS) for the final selection of studies.

\section{Eligibility criteria}

Studies that fulfil the following criteria were included. Firstly, studies that assessed and reported empirical data (primary or secondary) on the association between social support and depression, anxiety, or self-harm during pregnancy. Second, the types of study design are limited to observational studies such as cross-sectional, casecontrol, or cohort study design. Third, the participants of reported studies needed to be adult pregnant mothers whose age is 18 years old and above. Fourth, studies in which depression, anxiety and self-harm was confirmed by validated self-report screening instruments, structured interviews or other diagnostic criteria. The exclusion criteria's were as follows: (1) Studies like clinical trials, literature reviews, commentaries, short communications, and letters to the editor, (2) studies that failed to report tool used to confirm the presence of mental health problems (depression, anxiety and self-harm) and the tool used to measure the social support given for pregnant women and (3) studies not published in the English language.

\section{Definition of outcome variables}

In this study, mental health problems were operationalized as any diagnosed depressive disorders, general anxiety disorder, or suicidality (thoughts of self-harm, or suicidal attempt) according to standard diagnostic criteria such as the International Classification of Disease [46], the Diagnostic Statistical Manual of Mental Disorders (DSM) [47] or identified depressive symptoms or anxiety symptoms based on the valid screening tool.

\section{Definition of the exposure variable}

In the current study, social support is broadly defined as the provision of emotional (e.g. caring), or informational (e.g. notifying someone of important information) support, instrumental (e.g. helping with housekeeping), 
tangible (e.g. practical support like financial aid), and/or psychological support for somebody by the social network of family members, friends, or community members [48].

\section{Quality appraisal and methods of data extraction}

The modified version of the Newcastle-Ottawa Scale (NOS) was used to evaluate the methodologic quality (sample size, representativeness, comparability, nonresponse, ascertainment of outcome and statistical analysis) of the studies included in the current systematic review and meta-analysis [49]. Data extraction was independently completed from articles with good quality standards (NOS score $\geq 7$ points) by two investigators $(\mathrm{AB}, \mathrm{WP})$ [50]. During the review process, any disagreement between the two investigators $(\mathrm{AB}, \mathrm{WP})$ was resolved through continuous discussion with review team members until consensus was reached. A specific form of data extraction format prepared in the Microsoft Excel spreadsheet (Additional file 2) was used. The following information was extracted from eligible fulltext articles: author's name, year of publication, country, sample size, study design, type of support, source of support, instrument employed, study setting, and measure of association and confidence interval.

\section{Data synthesis method}

STATA IC version 16 statistical software was used to conduct a meta-analysis and estimate effect sizes. Studies were pooled to calculate pooled adjusted odds ratios and $95 \%$ CI using a random-effect model [51]. Adjusted odds ratios (AORs) were used as the preferred measure of association for meta-analysis, however, studies that analyse and report social support as a continuous exposure variable were reported in the narrative analysis. The narrative analysis was separately conducted for the association between social support and antenatal depression, antenatal anxiety, and antenatal self-harm. Among the studies included in the meta-analysis, most studies compared low social support with high/good social support. However, studies using low social support as a reference category were changed using the reciprocal method to maintain uniformity [52]. A meta-analysis of adjusted odds ratios for the association between low social support and outcome variables were calculated after logtransforming the estimates from eligible studies. If more than one outcome was reported from a single study each outcome was analysed independently.

Publication bias, heterogeneity, and subgroup analysis Possible publication bias was assessed through inspection of the funnel plot and Egger's regression tests [53, 54].
The results of the tests suggested the existence of possible publication bias ( $\mathrm{p}<0.05$ in Egger's test), the final effect size (POR) was determined using Duval and Tweedie's Trim and Fill analysis in the Random-effects model [55]. The trim and fill analysis is a non-parametric method for approximating the number of missing studies that might exist and helps in reducing and correcting publication bias in meta-analysis. The presence of heterogeneity between studies was assessed using $\mathrm{Q}$ and the $\mathrm{I}^{2}$ statistics [51]. The $\mathrm{I}^{2}$ provides an estimate of the percentage of the variability in effect estimates that is due to heterogeneity rather than sampling error or chance differences. $\mathrm{I}^{2}$ statistics range from 0 to $100 \%$ and values of 25,50 and $75 \%$ were considered to represent low, medium and high respectively [56]. A value of $0 \%$ indicates no observed heterogeneity while $100 \%$ indicates significant heterogeneity and a p-value $<0.05$ was used to declare significant heterogeneity [56]. The possible sources of heterogeneity were identified using a univariate meta-regression model. Sub-group analyses were conducted based on study design, study setting, economic level of countries (low, middle and high income), median sample size and publication year. Sensitivity analysis was also undertaken to examine the effect of a single study on the overall effect size.

\section{Result}

\section{Selection of studies}

As indicated in Fig. 1, during the search strategy, 3760 papers were retrieved from five electronic databases. Also, an additional six citations were identified through a manual search of reference lists. After 1624 duplicates were removed, preliminary screening of the titles and abstracts of 2142 articles was conducted, and as a result a further 1862 articles were excluded. The remaining 280 articles met the criteria for full-text review with another 213 articles being excluded. Finally, 67 articles fulfilled the inclusion criteria and were included in the current systematic review and meta-analysis.

\section{Study characteristics}

The characteristics of the included articles are presented in Table 1 . Of the studies included in the present systematic review and meta-analysis, 21,18 , and 16 studies were conducted in high, middle, and low-income countries respectively representing 64,449 pregnant women. The majority of the studies, 31(47\%), used the Edinburgh Postnatal Depression Scale (EPDS) for screening antenatal depression. The sample size of included studies ranges between 82 participants in the US [57] and 5337 participants in Canada [58]. 


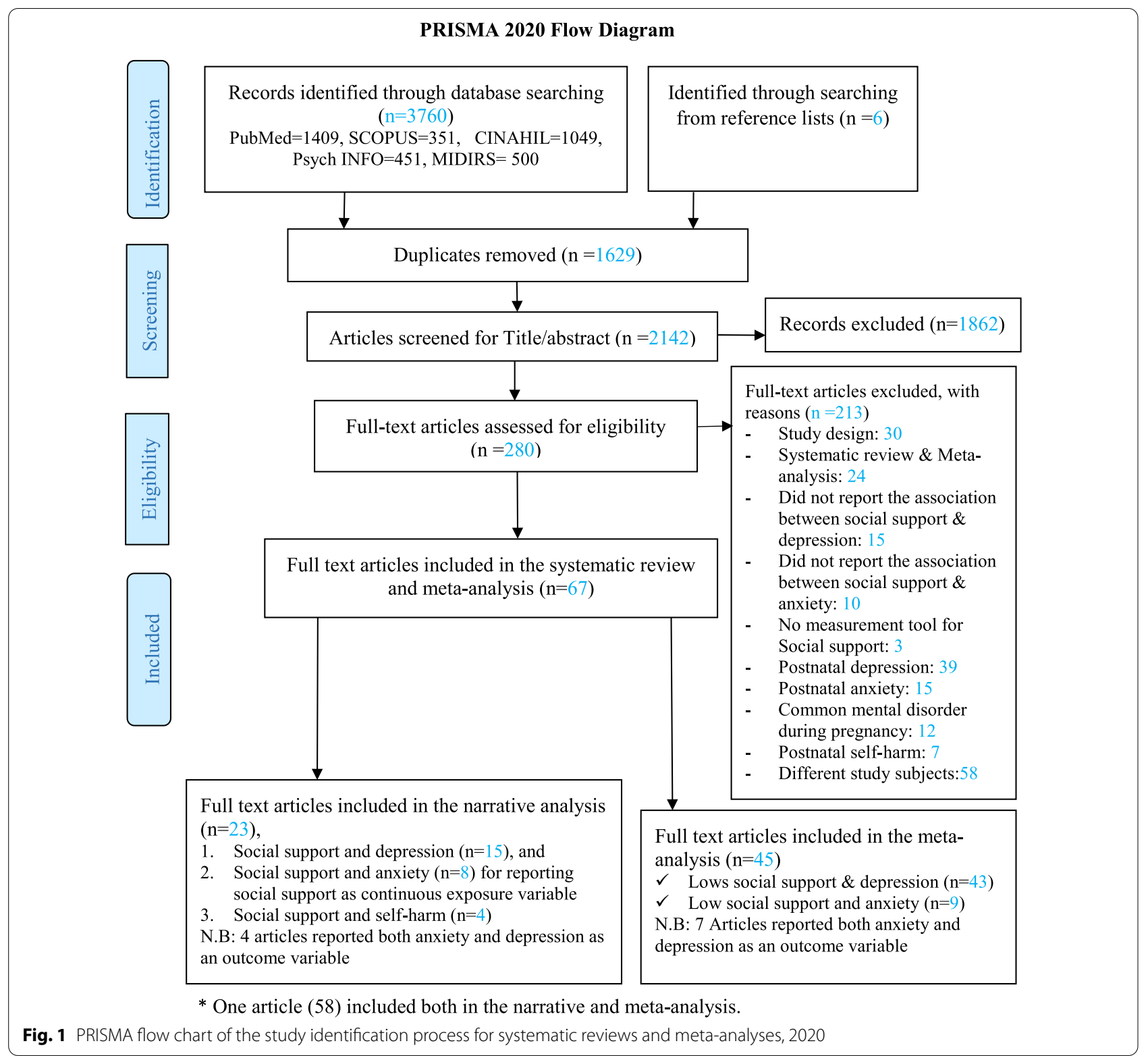

The articles included in the current systematic review and meta-analysis used 22 different valid measures of social support tools. From the total social support measures, the 3-item Oslo social support scale (OSSS-3) and Multidimensional Scale of Perceived Social Support (MSPSS) were the most dominant ones used by 11 and 10 studies respectively (Fig. 2). Details on the social support tools used and their reliability is outlined in Table 2.

Overall, from the total identified articles, 45 studies reported odds ratio (OR) as a measure of association between social support with antenatal depression and we included them in the meta-analysis presented here. Nine studies reported odds ratio (OR) as the measure of association between social support and antenatal anxiety and were included in the meta-analysis presented here. Twenty-three studies were included in the narrative analysis for analysing social support as a continuous variable $[39,59,62,72,77$, 79-82, 93, 99, 101, 106, 108, 110, 112-114]. Also, 4 studies $[43,89,119,120]$ that examined the relationship between self-harm and social support were included in the narrative analysis.

\section{Quality appraisal}

From the included 67 articles, all scored greater or equal to 7 out of 10 on the NOS which are thereby considered as 
Table1 Summary characteristics of studies investigating the association between social support and mental health problems (depression, anxiety and self-harm) during pregnancy ( $N=66$, from 2000-2019)

\begin{tabular}{|c|c|c|c|c|c|c|c|c|c|}
\hline \multirow[t]{2}{*}{ S. no. } & \multirow{2}{*}{$\begin{array}{l}\text { Author, } \\
\text { country, } \\
\text { publication } \\
\text { year }\end{array}$} & \multirow{2}{*}{$\begin{array}{l}\text { Type of } \\
\text { support }\end{array}$} & \multirow{2}{*}{$\begin{array}{l}\text { Source of } \\
\text { support }\end{array}$} & \multirow[t]{2}{*}{ Sample size } & \multirow[t]{2}{*}{ Setting } & \multirow{2}{*}{$\begin{array}{l}\text { Study } \\
\text { design }\end{array}$} & \multicolumn{2}{|c|}{ Measurement } & \multirow[t]{2}{*}{ Measure of association } \\
\hline & & & & & & & $\begin{array}{l}\text { Mental } \\
\text { health } \\
\text { problems }\end{array}$ & $\begin{array}{l}\text { Social } \\
\text { support }\end{array}$ & \\
\hline 1 & $\begin{array}{l}\text { Abujilban } \\
\text { SK., et al., } \\
\text { Jordan, } \\
2013 \text { [58] }\end{array}$ & $\begin{array}{l}\text { General sup- } \\
\text { port }\end{array}$ & $\begin{array}{l}\text { Family/non- } \\
\text { family }\end{array}$ & 218 & Facility & $\begin{array}{l}\text { Cross-sec- } \\
\text { tional }\end{array}$ & $\begin{array}{l}\text { Depression: } \\
\text { EPDS }\end{array}$ & $\begin{array}{l}\text { DUSOCS } \\
\text { (12 item) }\end{array}$ & $r=-0.022, P>0.05$ \\
\hline 2 & $\begin{array}{l}\text { Adewuya } \\
\text { AO., et al., } \\
\text { Nigeria, } \\
2007 \text { [59] }\end{array}$ & $\begin{array}{l}\text { Emotional/ } \\
\text { instrumen- } \\
\text { tal support }\end{array}$ & Partner & 181 & Facility & $\begin{array}{l}\text { Cross-sec- } \\
\text { tional }\end{array}$ & $\begin{array}{l}\text { Depression: } \\
\text { EPDS }\end{array}$ & PSSS & AOR: $6.08(1.42,26.04)$ \\
\hline 3 & $\begin{array}{l}\text { Agostini F., } \\
\text { et al., Italy, } \\
2015 \text { [60] }\end{array}$ & $\begin{array}{l}\text { General sup- } \\
\text { port }\end{array}$ & $\begin{array}{l}\text { Partner/fam- } \\
\text { ily/friend }\end{array}$ & 404 & Facility & Longitudinal & $\begin{array}{l}\text { Depression: } \\
\text { EPDS }\end{array}$ & MSPSS & AOR: $1.06(1.03,1.08)$ \\
\hline \multirow[t]{2}{*}{4} & \multirow{2}{*}{$\begin{array}{l}\text { Akiki S., et al., } \\
\text { UK, 2016 } \\
{[61]}\end{array}$} & \multirow{2}{*}{$\begin{array}{l}\text { General sup- } \\
\text { port }\end{array}$} & Family & \multirow[t]{2}{*}{1992} & \multirow[t]{2}{*}{ Facility } & \multirow[t]{2}{*}{ Longitudinal } & \multirow[t]{2}{*}{ Anxiety: STAI } & \multirow[t]{2}{*}{ PSS } & $\beta=-0.044, P=0.029$ \\
\hline & & & Partner & & & & & & $\beta=-0.033, P=0.0051$ \\
\hline 5 & $\begin{array}{l}\text { Anindyajati } \\
\text { G., et al., } \\
\text { Indonesia, } \\
2017 \text { [62] }\end{array}$ & $\begin{array}{l}\text { General sup- } \\
\text { port }\end{array}$ & $\begin{array}{l}\text { Partner/ } \\
\text { family }\end{array}$ & 107 & Community & $\begin{array}{l}\text { Cross-sec- } \\
\text { tional }\end{array}$ & $\begin{array}{l}\text { Depression: } \\
\text { LPGD }\end{array}$ & KD-24 item & AOR $0.21(0.05,0.84)$ \\
\hline \multirow[t]{2}{*}{6} & \multirow{2}{*}{$\begin{array}{l}\text { Bayrampour } \\
\text { H., et al., } \\
\text { Canada, } \\
2015 \text { [63] }\end{array}$} & \multirow[t]{2}{*}{$\begin{array}{l}\text { General sup- } \\
\text { port }\end{array}$} & \multirow[t]{2}{*}{$\begin{array}{l}\text { Family/part- } \\
\text { ner }\end{array}$} & \multirow[t]{2}{*}{3021} & \multirow[t]{2}{*}{ Facility } & \multirow[t]{2}{*}{ Longitudinal } & $\begin{array}{l}\text { Depression: } \\
\text { EPDS }\end{array}$ & \multirow[t]{2}{*}{$\begin{array}{l}\text { MOS-SSS (19 } \\
\text { item) }\end{array}$} & AOR $3.09(1.65,5.78)$ \\
\hline & & & & & & & Anxiety: STAI & & AOR $3.37(2.14,5.33)$ \\
\hline 7 & $\begin{array}{l}\text { Belay YB., } \\
\text { et al., } \\
\text { Ethiopia, } \\
2018 \text { [64] }\end{array}$ & $\begin{array}{l}\text { General sup- } \\
\text { port }\end{array}$ & Partner & 363 & Facility & $\begin{array}{l}\text { Cross-sec- } \\
\text { tional }\end{array}$ & $\begin{array}{l}\text { Depression: } \\
\text { BDI }\end{array}$ & MSSS & AOR $4.76(1.51,14.28)$ \\
\hline 8 & $\begin{array}{l}\text { Bernard } \\
\text { O., et al., } \\
\text { Jamaica, } \\
2018 \text { [65] }\end{array}$ & $\begin{array}{l}\text { Emotional } \\
\text { support }\end{array}$ & Partner & 3571 & Facility & Longitudinal & $\begin{array}{l}\text { Depression: } \\
\text { EPDS }\end{array}$ & MSPSS & AOR $3.14(1.69,5.84)$ \\
\hline 9 & $\begin{array}{l}\text { Biratu A., } \\
\text { et al., } \\
\text { Ethiopia, } \\
2015 \text { [66] }\end{array}$ & $\begin{array}{l}\text { General sup- } \\
\text { port }\end{array}$ & Partner & 422 & Facility & $\begin{array}{l}\text { Cross-sec- } \\
\text { tional }\end{array}$ & $\begin{array}{l}\text { Depression: } \\
\text { EPDS }\end{array}$ & PSS & AOR $1.89(1.06,3.35)$ \\
\hline 10 & $\begin{array}{l}\text { Bisetegn } \\
\text { TA., et al., } \\
\text { Ethiopia, } \\
2016 \text { [67] }\end{array}$ & $\begin{array}{l}\text { General sup- } \\
\text { port }\end{array}$ & $\begin{array}{l}\text { Family/Part- } \\
\text { ner }\end{array}$ & 527 & Community & $\begin{array}{l}\text { Cross-sec- } \\
\text { tional }\end{array}$ & $\begin{array}{l}\text { Depression: } \\
\text { EPDS }\end{array}$ & OSSS-3 & AOR $1.57(0.79,3.11)$ \\
\hline 11 & $\begin{array}{l}\text { Cankorur } \\
\text { vs., et al., } \\
\text { Turkey, } \\
2015 \text { [68] }\end{array}$ & $\begin{array}{c}\text { Emotional/ } \\
\text { practical } \\
\text { support }\end{array}$ & $\begin{array}{l}\text { Family/part- } \\
\text { ner }\end{array}$ & 730 & Facility & Longitudinal & $\begin{array}{l}\text { Depression: } \\
\text { EPDS }\end{array}$ & $\mathrm{CPQ}$ & AOR 1.07, $(1.01,1.15)$ \\
\hline 12 & $\begin{array}{l}\text { Chee C., } \\
\text { et al., } \\
\text { Singapore, } \\
2005 \text { [69] }\end{array}$ & $\begin{array}{l}\text { General sup- } \\
\text { port }\end{array}$ & $\begin{array}{l}\text { Family/part- } \\
\text { ner }\end{array}$ & 724 & Facility & Longitudinal & $\begin{array}{l}\text { Depression: } \\
\text { EPDS }\end{array}$ & $\begin{array}{l}\text { MOS-SSS } \\
\text { (19 item) }\end{array}$ & AOR $2.53(1.07-6.02)$ \\
\hline \multirow[t]{2}{*}{13} & \multirow{2}{*}{$\begin{array}{l}\text { Cheng E., } \\
\text { et al., USA, } \\
2016 \text { [70] }\end{array}$} & \multirow[t]{2}{*}{$\begin{array}{l}\text { General sup- } \\
\text { port }\end{array}$} & \multirow{2}{*}{$\begin{array}{l}\text { Friend/ } \\
\text { partner/ } \\
\text { relatives }\end{array}$} & 1764 & \multirow[t]{2}{*}{ Facility } & \multirow[t]{2}{*}{ Longitudinal } & $\begin{array}{l}\text { Depression: } \\
\text { EPDS }\end{array}$ & PSS & $\begin{array}{l}\text { AOR: } 3.1(1.7,5.7) \text { (Project } \\
\text { viva) }\end{array}$ \\
\hline & & & & 877 & & & Anxiety: STAI & & $\begin{array}{l}\text { AOR: } 1.9(1.1,3.3) \text { (Project } \\
\text { access) }\end{array}$ \\
\hline \multirow[t]{2}{*}{14} & \multirow{2}{*}{$\begin{array}{l}\text { Clements } \\
\text { AD., et al., } \\
\text { USA, } 2016 \\
\text { [71] }\end{array}$} & \multirow[t]{2}{*}{$\begin{array}{l}\text { General sup- } \\
\text { port }\end{array}$} & $\begin{array}{l}\text { Family/part- } \\
\text { ner }\end{array}$ & 106 & Facility & Longitudinal & $\begin{array}{l}\text { Depression: } \\
\text { CESD }\end{array}$ & PPP & $\begin{array}{l}\beta=-0.44, P<0.001 \\
\left(1^{s t} \text { trimester }\right)\end{array}$ \\
\hline & & & & & & & & & $\begin{array}{l}\beta=-0.33, P<0.001 \\
\text { (2nd trimester) }\end{array}$ \\
\hline
\end{tabular}


Table1 (continued)

\begin{tabular}{|c|c|c|c|c|c|c|c|c|c|}
\hline \multirow[t]{2}{*}{ S. no. } & \multirow{2}{*}{$\begin{array}{l}\text { Author, } \\
\text { country, } \\
\text { publication } \\
\text { year }\end{array}$} & \multirow{2}{*}{$\begin{array}{l}\text { Type of } \\
\text { support }\end{array}$} & \multirow{2}{*}{$\begin{array}{l}\text { Source of } \\
\text { support }\end{array}$} & \multirow[t]{2}{*}{ Sample size } & \multirow[t]{2}{*}{ Setting } & \multirow{2}{*}{$\begin{array}{l}\text { Study } \\
\text { design }\end{array}$} & \multicolumn{2}{|c|}{ Measurement } & \multirow[t]{2}{*}{ Measure of association } \\
\hline & & & & & & & $\begin{array}{l}\text { Mental } \\
\text { health } \\
\text { problems }\end{array}$ & $\begin{array}{l}\text { Social } \\
\text { support }\end{array}$ & \\
\hline 15 & $\begin{array}{l}\text { Dibaba } \\
\text { Y., et al., } \\
\text { Ethiopia, } \\
2013 \text { [72] }\end{array}$ & $\begin{array}{l}\text { General sup- } \\
\text { port }\end{array}$ & $\begin{array}{l}\text { Family/part- } \\
\text { ner }\end{array}$ & 627 & Community & $\begin{array}{l}\text { Cross-sec- } \\
\text { tional }\end{array}$ & $\begin{array}{l}\text { Depression: } \\
\text { EPDS }\end{array}$ & MSSS & AOR $4.34(2.12,9.1)$ \\
\hline 16 & $\begin{array}{l}\text { Dong X., } \\
\text { et al., } \\
\text { China, } \\
2013 \text { [73] }\end{array}$ & $\begin{array}{l}\text { General sup- } \\
\text { port }\end{array}$ & $\begin{array}{l}\text { Partner } \\
\text { Parents } \\
\text { Parents-in- } \\
\text { law }\end{array}$ & 520 & Facility & $\begin{array}{l}\text { Cross-sec- } \\
\text { tional }\end{array}$ & $\begin{array}{l}\text { Depression: } \\
\text { EPDS }\end{array}$ & OSSS-3 & $\begin{array}{l}\text { AOR: } 1.75 \text { (0.16-19.28), } \\
\text { AOR: } 0.56 \text { (0.06-5.13), } \\
\text { AOR: } 0.74(0.24-2.23)\end{array}$ \\
\hline 17 & $\begin{array}{l}\text { Dudas R., } \\
\text { et al., } \\
\text { Hungary, } \\
2012[74]\end{array}$ & $\begin{array}{l}\text { General sup- } \\
\text { port }\end{array}$ & Partner & 1719 & Community & $\begin{array}{l}\text { Cross-sec- } \\
\text { tional }\end{array}$ & $\begin{array}{l}\text { Depression: } \\
\text { LQ }\end{array}$ & MSPSS & AOR: 1.79(1.32-1.89) \\
\hline 18 & $\begin{array}{l}\text { Duko B., } \\
\text { et al., } \\
\text { Ethiopia, } \\
2019 \text { [75] }\end{array}$ & $\begin{array}{l}\text { General sup- } \\
\text { port }\end{array}$ & $\begin{array}{l}\text { Partner/ } \\
\text { parents/ } \\
\text { parents-in- } \\
\text { law }\end{array}$ & 317 & Facility & $\begin{array}{l}\text { Cross-sec- } \\
\text { tional }\end{array}$ & $\begin{array}{l}\text { Depression: } \\
\text { EPDS }\end{array}$ & OSSS-3 & AOR: $2.14(1.49,3.11)$ \\
\hline 19 & $\begin{array}{l}\text { Schetter } \\
\text { DC., et al., } \\
\text { Canada, } \\
2016 \text { [76] }\end{array}$ & $\begin{array}{l}\text { General sup- } \\
\text { port }\end{array}$ & $\begin{array}{l}\text { Family/part- } \\
\text { ner }\end{array}$ & 5271 & Facility & Longitudinal & Anxiety: BIPS & $\begin{array}{l}\text { MOS-SSS (19 } \\
\text { item) }\end{array}$ & $\begin{array}{l}\beta=0.08(0.01,0.15) \\
P>0.05\end{array}$ \\
\hline 20 & $\begin{array}{l}\text { Fall A., et al., } \\
\text { Canada, } \\
2013[57]\end{array}$ & $\begin{array}{l}\text { General sup- } \\
\text { port }\end{array}$ & Family & 5337 & Facility & Longitudinal & $\begin{array}{l}\text { Depression: } \\
\text { CES-D }\end{array}$ & ASSI & AOR: 4.47 (3.55-5.63) \\
\hline 21 & $\begin{array}{l}\text { Gao L., et al., } \\
\text { China, } \\
2019[77]\end{array}$ & $\begin{array}{l}\text { Emotional/ } \\
\text { Instrumen- } \\
\text { tal support }\end{array}$ & Partner & 278 & Facility & $\begin{array}{l}\text { Cross-sec- } \\
\text { tional }\end{array}$ & $\begin{array}{l}\text { Anxiety: SAS } \\
\text { Depression: } \\
\text { EPDS }\end{array}$ & PSSS & $\begin{array}{l}\text { AOR: } 2.86(1.70,4.83) \\
\text { AOR: } 2.56(1.52,4.30)\end{array}$ \\
\hline 22 & $\begin{array}{l}\text { Golbasi Z., } \\
\text { et al., Tur- } \\
\text { key, } 2010 \\
{[78]}\end{array}$ & $\begin{array}{l}\text { Emotional/ } \\
\text { Instrumen- } \\
\text { tal support }\end{array}$ & $\begin{array}{l}\text { Family/part- } \\
\text { ner/friend }\end{array}$ & 258 & Facility & $\begin{array}{l}\text { Cross-sec- } \\
\text { tional }\end{array}$ & $\begin{array}{l}\text { Depression: } \\
\text { EPDS }\end{array}$ & MSPSS & $r=-0.43 ; P<0.001$ \\
\hline 23 & $\begin{array}{l}\text { Gourounti } \\
\text { K., et al., } \\
\text { Greece, } \\
2013 \text { [79] }\end{array}$ & $\begin{array}{l}\text { General sup- } \\
\text { port }\end{array}$ & $\begin{array}{l}\text { Family/ } \\
\text { friends/ } \\
\text { partner }\end{array}$ & 165 & Facility & $\begin{array}{l}\text { Cross-sec- } \\
\text { tional }\end{array}$ & Anxiety: STAl & SSQ-6 & $\beta=0.131(0.19,2.37)$ \\
\hline 24 & $\begin{array}{l}\text { Hain S., et al., } \\
\text { Germany, } \\
2016[80]\end{array}$ & $\begin{array}{l}\text { Emotional/ } \\
\text { instrumen- } \\
\text { tal support }\end{array}$ & $\begin{array}{l}\text { Family/part- } \\
\text { ner/non- } \\
\text { family }\end{array}$ & 297 & Facility & Longitudinal & $\begin{array}{l}\text { Depression: } \\
\text { EPDS }\end{array}$ & F-SozU K-14 & $r=-0.45, p<0.01$ \\
\hline 25 & $\begin{array}{l}\text { Herbell K., } \\
\text { et al., USA, } \\
2019 \text { [81] }\end{array}$ & $\begin{array}{l}\text { General sup- } \\
\text { port }\end{array}$ & $\begin{array}{l}\text { Family/part- } \\
\text { ner }\end{array}$ & 82 & Facility & $\begin{array}{l}\text { Cross-sec- } \\
\text { tional }\end{array}$ & $\begin{array}{l}\text { Depression: } \\
\text { CESD }\end{array}$ & $\begin{array}{l}\text { MOS-SSS (19 } \\
\text { item) }\end{array}$ & $\beta=-0.751, P<0.001$ \\
\hline 26 & $\begin{array}{l}\text { Heyningen } \\
\text { T., et al., } \\
\text { South } \\
\text { Africa, } \\
2017 \text { [82] }\end{array}$ & $\begin{array}{l}\text { Emotional/ } \\
\text { instrumen- } \\
\text { tal support }\end{array}$ & Friends & 376 & Facility & $\begin{array}{l}\text { Cross-sec- } \\
\text { tional }\end{array}$ & $\begin{array}{l}\text { Anxiety: } \\
\text { MINI }\end{array}$ & MSPSS & AOR: $1.05(1.01,1.09)$ \\
\hline 27 & $\begin{array}{l}\text { Jeong } \mathrm{H}_{.,} \\
\text {et al., } \\
\text { South } \\
\text { Africa, } \\
2013 \text { [83] }\end{array}$ & $\begin{array}{c}\text { Emotional } \\
\text { support }\end{array}$ & Mother & 1262 & Facility & $\begin{array}{l}\text { Cross-sec- } \\
\text { tional }\end{array}$ & $\begin{array}{l}\text { Depression: } \\
\text { EPDS }\end{array}$ & MSPSS & AOR: $1.5(1.31-1.71)$ \\
\hline 28 & $\begin{array}{l}\text { Lau Y., et al., } \\
\text { China, } \\
2011[84]\end{array}$ & $\begin{array}{l}\text { Emotional/ } \\
\text { tangible } \\
\text { support }\end{array}$ & Family & 1609 & Facility & $\begin{array}{l}\text { Cross-sec- } \\
\text { tional }\end{array}$ & $\begin{array}{l}\text { Depression: } \\
\text { EPDS }\end{array}$ & ISEL & AOR: $1.9(1.582,2.520)$ \\
\hline
\end{tabular}


Table1 (continued)

\begin{tabular}{|c|c|c|c|c|c|c|c|c|c|}
\hline \multirow[t]{2}{*}{ S. no. } & \multirow{2}{*}{$\begin{array}{l}\text { Author, } \\
\text { country, } \\
\text { publication } \\
\text { year }\end{array}$} & \multirow{2}{*}{$\begin{array}{l}\text { Type of } \\
\text { support }\end{array}$} & \multirow{2}{*}{$\begin{array}{l}\text { Source of } \\
\text { support }\end{array}$} & \multirow[t]{2}{*}{ Sample size } & \multirow[t]{2}{*}{ Setting } & \multirow{2}{*}{$\begin{array}{l}\text { Study } \\
\text { design }\end{array}$} & \multicolumn{2}{|c|}{ Measurement } & \multirow[t]{2}{*}{ Measure of association } \\
\hline & & & & & & & $\begin{array}{l}\text { Mental } \\
\text { health } \\
\text { problems }\end{array}$ & $\begin{array}{l}\text { Social } \\
\text { support }\end{array}$ & \\
\hline \multirow[t]{2}{*}{29} & \multirow{2}{*}{$\begin{array}{l}\text { Lee AM., } \\
\text { et al., Hong } \\
\text { Kong, } 2007 \\
{[85]}\end{array}$} & \multirow{2}{*}{$\begin{array}{l}\text { Emotional/ } \\
\text { instrumen- } \\
\text { tal support }\end{array}$} & \multirow{2}{*}{ Partner } & \multirow[t]{2}{*}{357} & \multirow[t]{2}{*}{ Facility } & \multirow[t]{2}{*}{ Longitudinal } & $\begin{array}{c}\text { Anxiety: } \\
\text { HADS }\end{array}$ & \multirow[t]{2}{*}{ PSSS } & AOR: $1.72(1.05,2.85)$ \\
\hline & & & & & & & $\begin{array}{l}\text { Depression: } \\
\text { HADS }\end{array}$ & & AOR: $1.69(1.01,2.85)$ \\
\hline 30 & $\begin{array}{l}\text { LiY., et al., } \\
\text { China, } \\
2017 \text { [86] }\end{array}$ & $\begin{array}{l}\text { Emotional/ } \\
\text { instrumen- } \\
\text { tal support }\end{array}$ & Partner & 240 & Facility & Longitudinal & $\begin{array}{l}\text { Depression: } \\
\text { EPDS }\end{array}$ & PSSS & AOR $0.99(0.94,1.05)$ \\
\hline 31 & $\begin{array}{l}\text { Nath A.,et al., } \\
\text { India, } 2019 \\
\text { [87] }\end{array}$ & $\begin{array}{l}\text { Emotional/ } \\
\text { instrumen- } \\
\text { tal support }\end{array}$ & $\begin{array}{l}\text { Family/part- } \\
\text { ner }\end{array}$ & 380 & Facility & $\begin{array}{l}\text { Cross-sec- } \\
\text { tional }\end{array}$ & Anxiety: PRT & MSPSS & AOR: $1.76(1.04,2.98)$ \\
\hline 32 & $\begin{array}{l}\text { Onah MN., } \\
\text { et al., } \\
\text { South } \\
\text { Africa, } \\
2016 \text { [88] }\end{array}$ & $\begin{array}{l}\text { Emotional/ } \\
\text { instrumen- } \\
\text { tal support }\end{array}$ & $\begin{array}{l}\text { Family/ } \\
\text { friends/ } \\
\text { partner }\end{array}$ & 376 & Facility & $\begin{array}{l}\text { Cross-sec- } \\
\text { tional }\end{array}$ & $\begin{array}{l}\text { Self-Harm: } \\
\text { SIB }\end{array}$ & MSPSS & AOR: $1.07(1.01,1.15)$ \\
\hline 33 & $\begin{array}{l}\text { Pajulo M., } \\
\text { et al., Fin- } \\
\text { land, } 2001 \\
\text { [89] }\end{array}$ & $\begin{array}{l}\text { General sup- } \\
\text { port }\end{array}$ & $\begin{array}{l}\text { Partner/ } \\
\text { parents/ } \\
\text { mother } \\
\text { in-law/ } \\
\text { friend }\end{array}$ & 391 & Facility & $\begin{array}{l}\text { Cross-sec- } \\
\text { tional }\end{array}$ & $\begin{array}{l}\text { Depression: } \\
\text { EPDS }\end{array}$ & SSQ-12 & AOR: $4.2(0.9,20.2)$ \\
\hline 34 & $\begin{array}{l}\text { Rashid A., } \\
\text { et al., } \\
\text { Malaysia, } \\
2017 \text { [90] }\end{array}$ & $\begin{array}{l}\text { General sup- } \\
\text { port }\end{array}$ & $\begin{array}{l}\text { Partner/ } \\
\text { parents/ } \\
\text { parents-in- } \\
\text { law }\end{array}$ & 3000 & Facility & $\begin{array}{l}\text { Cross-sec- } \\
\text { tional }\end{array}$ & $\begin{array}{l}\text { Depression: } \\
\text { EPDS }\end{array}$ & OSSS-3 & AOR: $2.16(1.77,2.64)$ \\
\hline 35 & $\begin{array}{l}\text { Rubertsson } \\
\text { C.et al., } \\
\text { Sweden, } \\
2003 \text { [91] }\end{array}$ & $\begin{array}{l}\text { General sup- } \\
\text { port }\end{array}$ & $\begin{array}{l}\text { Partner/ } \\
\text { parents/ } \\
\text { parents-in- } \\
\text { law }\end{array}$ & 608 & Facility & Longitudinal & $\begin{array}{l}\text { Depression: } \\
\text { EPDS }\end{array}$ & OSSS-3 & AOR: $6.9(3.4,13.9)$ \\
\hline \multirow[t]{2}{*}{36} & \multirow{2}{*}{$\begin{array}{l}\text { Shafaie FS., } \\
\text { et al., Iran, } \\
2017 \text { [92] }\end{array}$} & \multirow[t]{2}{*}{$\begin{array}{l}\text { General sup- } \\
\text { port }\end{array}$} & \multirow[t]{2}{*}{$\begin{array}{l}\text { Family/Part- } \\
\text { ner }\end{array}$} & \multirow[t]{2}{*}{372} & \multirow[t]{2}{*}{ Facility } & \multirow[t]{2}{*}{$\begin{array}{l}\text { Cross-sec- } \\
\text { tional }\end{array}$} & $\begin{array}{l}\text { Anxiety: } \\
\text { DASS }\end{array}$ & \multirow[t]{2}{*}{ PRQ- 85} & $r=-0.456, p<.001$ \\
\hline & & & & & & & $\begin{array}{l}\text { Depression: } \\
\text { DASS }\end{array}$ & & $r=-0.642, p<.001$ \\
\hline 37 & $\begin{array}{l}\text { Sheeba B., } \\
\text { et al., India, } \\
2019 \text { [93] }\end{array}$ & $\begin{array}{l}\text { Emotional/ } \\
\text { instrumen- } \\
\text { tal support }\end{array}$ & $\begin{array}{l}\text { Family/ } \\
\text { friends/ } \\
\text { partner }\end{array}$ & 280 & Facility & Longitudinal & $\begin{array}{l}\text { Depression: } \\
\text { EPDS }\end{array}$ & MSPSS & AOR: $1.785(0.915,3.48)$ \\
\hline 38 & $\begin{array}{l}\text { Sidebot- } \\
\text { tom AC., } \\
\text { et al., USA, } \\
2017[94]\end{array}$ & $\begin{array}{l}\text { General sup- } \\
\text { port }\end{array}$ & $\begin{array}{l}\text { Family/ } \\
\text { friend }\end{array}$ & 2341 & Facility & Longitudinal & $\begin{array}{l}\text { Depression: } \\
\text { PHQ-9 }\end{array}$ & MSSI & AOR: $1.85(1.31,2.60)$ \\
\hline 39 & $\begin{array}{l}\text { Spoozak L., } \\
\text { et al., USA, } \\
2008 \text { [95] }\end{array}$ & $\begin{array}{l}\text { Emotional/ } \\
\text { instrumen- } \\
\text { tal support }\end{array}$ & $\begin{array}{l}\text { Mother/ } \\
\text { partner }\end{array}$ & 783 & Facility & $\begin{array}{l}\text { Cross-sec- } \\
\text { tional }\end{array}$ & $\begin{array}{l}\text { Depression: } \\
\text { CIDI }\end{array}$ & MKSSI & AOR: $2.39(1.63,3.52)$ \\
\hline 40 & $\begin{array}{l}\text { Stewart } \\
\text { RC., et al., } \\
\text { Malawi, } \\
2014 \text { [96] }\end{array}$ & $\begin{array}{l}\text { Emotional/ } \\
\text { instrumen- } \\
\text { tal support }\end{array}$ & $\begin{array}{l}\text { Family/part- } \\
\text { ner/friend }\end{array}$ & 503 & Facility & $\begin{array}{l}\text { Cross-sec- } \\
\text { tional }\end{array}$ & $\begin{array}{l}\text { Depression: } \\
\text { DSM IV }\end{array}$ & MSPSS & AOR $1.11(1.04,1.17)$ \\
\hline \multirow[t]{2}{*}{41} & \multirow{2}{*}{$\begin{array}{l}\text { Xian T., et al., } \\
\text { China, } \\
2019 \text { [97] }\end{array}$} & \multirow[t]{2}{*}{$\begin{array}{l}\text { General sup- } \\
\text { port }\end{array}$} & \multirow[t]{2}{*}{$\begin{array}{l}\text { Family/part- } \\
\text { ner }\end{array}$} & \multirow[t]{2}{*}{1220} & \multirow[t]{2}{*}{ Facility } & \multirow[t]{2}{*}{ Longitudinal } & $\begin{array}{l}\text { Anxiety: } \\
\text { HAMA }\end{array}$ & SSRS-10 & AOR: $5.09(2.41,10.77)$ \\
\hline & & & & & & & $\begin{array}{l}\text { Depression: } \\
\text { SDS }\end{array}$ & & AOR $3.18(1.46,6.96)$ \\
\hline 42 & $\begin{array}{l}\text { Verreault } \\
\text { N., et al., } \\
\text { Canada, } \\
2014 \text { [98] }\end{array}$ & $\begin{array}{l}\text { General sup- } \\
\text { port }\end{array}$ & $\begin{array}{l}\text { Family/ } \\
\text { partner/ } \\
\text { friends }\end{array}$ & 364 & Facility & $\begin{array}{l}\text { Cross-sec- } \\
\text { tional }\end{array}$ & $\begin{array}{l}\text { Depression: } \\
\text { EPDS }\end{array}$ & $\begin{array}{l}\text { MOS-SSS (19 } \\
\text { item) }\end{array}$ & $\beta:-0.32, P<0.001$ \\
\hline
\end{tabular}


Table1 (continued)

\begin{tabular}{|c|c|c|c|c|c|c|c|c|c|}
\hline \multirow[t]{2}{*}{ S. no. } & \multirow{2}{*}{$\begin{array}{l}\text { Author, } \\
\text { country, } \\
\text { publication } \\
\text { year }\end{array}$} & \multirow{2}{*}{$\begin{array}{l}\text { Type of } \\
\text { support }\end{array}$} & \multirow{2}{*}{$\begin{array}{l}\text { Source of } \\
\text { support }\end{array}$} & \multirow[t]{2}{*}{ Sample size } & \multirow[t]{2}{*}{ Setting } & \multirow{2}{*}{$\begin{array}{l}\text { Study } \\
\text { design }\end{array}$} & \multicolumn{2}{|l|}{ Measurement } & \multirow[t]{2}{*}{ Measure of association } \\
\hline & & & & & & & $\begin{array}{l}\text { Mental } \\
\text { health } \\
\text { problems }\end{array}$ & $\begin{array}{l}\text { Social } \\
\text { support }\end{array}$ & \\
\hline 43 & $\begin{array}{l}\text { Woldetensay } \\
\text { YK.,et al., } \\
\text { Ethiopia, } \\
2018 \text { [99] }\end{array}$ & $\begin{array}{l}\text { General sup- } \\
\text { port }\end{array}$ & $\begin{array}{l}\text { Family/ } \\
\text { friends/ } \\
\text { partner }\end{array}$ & 4680 & Community & Longitudinal & $\begin{array}{l}\text { Depression: } \\
\text { PHQ-9 }\end{array}$ & MSSS & AOR: $1.63(1.31-2.02)$ \\
\hline 44 & $\begin{array}{l}\text { Yanikkerem } \\
\text { E., et al., } \\
\text { Turkey, } \\
2013 \text { [100] }\end{array}$ & $\begin{array}{l}\text { Emotional/ } \\
\text { instrumen- } \\
\text { tal support }\end{array}$ & Partner & 651 & Facility & $\begin{array}{l}\text { Cross-sec- } \\
\text { tional }\end{array}$ & $\begin{array}{l}\text { Depression: } \\
\text { BDI }\end{array}$ & PSSS & $\beta=2.42,(0.707,4.135)$ \\
\hline 45 & $\begin{array}{l}\text { Zeng Y., } \\
\text { et al., } \\
\text { China, } \\
2015 \text { [101] }\end{array}$ & $\begin{array}{l}\text { Emotional/ } \\
\text { instrumen- } \\
\text { tal support }\end{array}$ & $\begin{array}{l}\text { Family/part- } \\
\text { ner/friend }\end{array}$ & 292 & Facility & $\begin{array}{l}\text { Cross-sec- } \\
\text { tional }\end{array}$ & $\begin{array}{l}\text { Depression: } \\
\text { SDS }\end{array}$ & SSRS-10 & AOR $1.08(1.03,1.13)$ \\
\hline 46 & $\begin{array}{l}\text { Sahile MA., } \\
\text { et al., } \\
\text { Ethiopia, } \\
2017 \text { [102] }\end{array}$ & $\begin{array}{l}\text { General sup- } \\
\text { port }\end{array}$ & $\begin{array}{l}\text { Partner/ } \\
\text { parents/ } \\
\text { parents-in- } \\
\text { law }\end{array}$ & 231 & Facility & $\begin{array}{l}\text { Cross-sec- } \\
\text { tional }\end{array}$ & $\begin{array}{l}\text { Depression: } \\
\text { BDI }\end{array}$ & OSSS-3 & AOR: $2.63(0.34,20$ \\
\hline 47 & $\begin{array}{l}\text { Records C., } \\
\text { et al., USA, } \\
2007 \text { [103] }\end{array}$ & $\begin{array}{l}\text { Emotional/ } \\
\text { instrumen- } \\
\text { tal support }\end{array}$ & $\begin{array}{l}\text { Family/ } \\
\text { friends/ } \\
\text { partner }\end{array}$ & 139 & Facility & $\begin{array}{l}\text { Cross-sec- } \\
\text { tional }\end{array}$ & $\begin{array}{l}\text { Depression: } \\
\text { CESD }\end{array}$ & MSPSS & $\beta=1.64, P<0.001$ \\
\hline 48 & $\begin{array}{l}\text { Marchesi C., } \\
\text { et al., Italy, } \\
2014 \text { [104] }\end{array}$ & $\begin{array}{l}\text { General sup- } \\
\text { port }\end{array}$ & $\begin{array}{l}\text { Family/ } \\
\text { friends/ } \\
\text { partner }\end{array}$ & 277 & Facility & Longitudinal & $\begin{array}{l}\text { Anxiety: } \\
\text { HADS }\end{array}$ & ASSI & AOR: $4.2(1.1,15.5)$ \\
\hline \multirow[t]{2}{*}{49} & \multirow{2}{*}{$\begin{array}{l}\text { Waqas A., } \\
\text { et al., Paki- } \\
\text { stan, 2015 } \\
\text { [105] }\end{array}$} & \multirow[t]{2}{*}{$\begin{array}{l}\text { General sup- } \\
\text { port }\end{array}$} & \multirow{2}{*}{$\begin{array}{l}\text { Family/ } \\
\text { friend/ } \\
\text { partner/oth- } \\
\text { ers }\end{array}$} & \multirow[t]{2}{*}{500} & \multirow[t]{2}{*}{ Facility } & \multirow[t]{2}{*}{$\begin{array}{l}\text { Cross-sec- } \\
\text { tional }\end{array}$} & $\begin{array}{l}\text { Anxiety- } \\
\text { HADS }\end{array}$ & \multirow[t]{2}{*}{ SPS } & $r=-0.433, P<0.001$ \\
\hline & & & & & & & $\begin{array}{l}\text { Depression- } \\
\text { HADS }\end{array}$ & & $r=-0.453, P<0.001$ \\
\hline 50 & $\begin{array}{l}\text { Westdahl C., } \\
\text { et al., USA, } \\
2007 \text { [106] }\end{array}$ & $\begin{array}{l}\text { General sup- } \\
\text { port }\end{array}$ & $\begin{array}{l}\text { Family/par- } \\
\text { ent }\end{array}$ & 1047 & Facility & Longitudinal & $\begin{array}{l}\text { Depression: } \\
\text { CESD }\end{array}$ & SSRS-10 & AOR: $2.29(1.21,4.33)$ \\
\hline \multirow[t]{2}{*}{51} & \multirow{2}{*}{$\begin{array}{l}\text { Nasreen } \\
\text { HE., et al., } \\
\text { Bangla- } \\
\text { desh, } 2011 \\
\text { [107] }\end{array}$} & \multirow[t]{2}{*}{$\begin{array}{l}\text { General sup- } \\
\text { port }\end{array}$} & \multirow{2}{*}{$\begin{array}{l}\text { Partner/ } \\
\text { parents/ } \\
\text { parents-in- } \\
\text { law }\end{array}$} & \multirow[t]{2}{*}{720} & \multirow[t]{2}{*}{ Community } & \multirow[t]{2}{*}{ Longitudinal } & $\begin{array}{l}\text { Depression: } \\
\text { EPDS }\end{array}$ & \multirow[t]{2}{*}{ OSSS-3 } & AOR: $2.23(2.12,3.62)$ \\
\hline & & & & & & & Anxiety: STAI & & $\beta:-1.1447, P<0.05$ \\
\hline 52 & $\begin{array}{l}\text { Leigh B., } \\
\text { et al., } \\
\text { Australia, } \\
2008 \text { [39] }\end{array}$ & $\begin{array}{l}\text { General sup- } \\
\text { port }\end{array}$ & $\begin{array}{l}\text { Family/ } \\
\text { friend/ } \\
\text { partner/ } \\
\text { others }\end{array}$ & 367 & Facility & Longitudinal & $\begin{array}{l}\text { Depression: } \\
\text { BDI }\end{array}$ & SPS & $\beta=-0.18, P<0.001$ \\
\hline \multirow[t]{2}{*}{53} & \multirow{2}{*}{$\begin{array}{l}\text { Martini } \\
\text { J., et al., } \\
\text { Germany, } \\
2015 \text { [108] }\end{array}$} & \multirow[t]{2}{*}{$\begin{array}{l}\text { General sup- } \\
\text { port }\end{array}$} & \multirow{2}{*}{$\begin{array}{l}\text { Family/ } \\
\text { friends/ } \\
\text { partner }\end{array}$} & \multirow[t]{2}{*}{306} & \multirow[t]{2}{*}{ Community } & \multirow[t]{2}{*}{ Longitudinal } & $\begin{array}{l}\text { Anxiety: } \\
\text { CIDI-V }\end{array}$ & \multirow[t]{2}{*}{ SSQ-12 } & AOR: $2.27(1.42,3.70)$ \\
\hline & & & & & & & $\begin{array}{l}\text { Depression: } \\
\text { CIDIV }\end{array}$ & & AOR: $2.43,(1.19,5)$ \\
\hline 54 & $\begin{array}{l}\text { Rubertsson } \\
\text { C. et al., } \\
\text { Sweden, } \\
2003 \text { [91] }\end{array}$ & $\begin{array}{l}\text { General sup- } \\
\text { port }\end{array}$ & $\begin{array}{l}\text { Partner/ } \\
\text { parent/ } \\
\text { parents-in- } \\
\text { law }\end{array}$ & 3011 & Facility & Longitudinal & $\begin{array}{l}\text { Depression: } \\
\text { EPDS }\end{array}$ & OSSS-3 & AOR: $4.4(2.7,7.4)$ \\
\hline \multirow[t]{2}{*}{55} & Huang M., & General sup- & Family/ & 158 & Facility & Longitudinal & Anxiety: STAI & MSSS & $\beta=-0.79(-1.16,-0.42)$ \\
\hline & $\begin{array}{l}\text { et al., Tai- } \\
\text { wan, } 2019 \\
\text { [109] }\end{array}$ & port & $\begin{array}{l}\text { friends/ } \\
\text { partner }\end{array}$ & & & & $\begin{array}{l}\text { Depression: } \\
\text { EPDS }\end{array}$ & & $\begin{array}{l}\beta=-0.44(-0.63 \\
\quad-0.24)\end{array}$ \\
\hline 56 & $\begin{array}{l}\text { Jesse ED., } \\
\text { et I, USA, } \\
2005[110]\end{array}$ & $\begin{array}{l}\text { General sup- } \\
\text { port }\end{array}$ & $\begin{array}{l}\text { Partner/oth- } \\
\text { ers }\end{array}$ & 130 & Facility & $\begin{array}{l}\text { Cross-sec- } \\
\text { tional }\end{array}$ & $\begin{array}{l}\text { Depression: } \\
\text { BDI }\end{array}$ & PPP & AOR:1 $(0.98,1.02), \mathrm{P}>0.05$ \\
\hline 57 & $\begin{array}{l}\text { Blaney NT., } \\
\text { et al., USA, } \\
2004 \text { [111] }\end{array}$ & $\begin{array}{l}\text { General sup- } \\
\text { port }\end{array}$ & $\begin{array}{l}\text { Friend/ } \\
\text { partner/ } \\
\text { relatives }\end{array}$ & 325 & Facility & $\begin{array}{l}\text { Cross-sec- } \\
\text { tional }\end{array}$ & $\begin{array}{l}\text { Depression: } \\
\text { CESD }\end{array}$ & PSS & $r=-0.25, P<0.001$ \\
\hline
\end{tabular}


Table1 (continued)

\begin{tabular}{|c|c|c|c|c|c|c|c|c|c|}
\hline \multirow{2}{*}{ S. no. } & \multirow{2}{*}{$\begin{array}{l}\text { Author, } \\
\text { country, } \\
\text { publication } \\
\text { year }\end{array}$} & \multirow{2}{*}{$\begin{array}{l}\text { Type of } \\
\text { support }\end{array}$} & \multirow{2}{*}{$\begin{array}{l}\text { Source of } \\
\text { support }\end{array}$} & \multirow[t]{2}{*}{ Sample size } & \multirow[t]{2}{*}{ Setting } & \multirow{2}{*}{$\begin{array}{l}\text { Study } \\
\text { design }\end{array}$} & \multicolumn{2}{|c|}{ Measurement } & \multirow[t]{2}{*}{ Measure of association } \\
\hline & & & & & & & $\begin{array}{l}\text { Mental } \\
\text { health } \\
\text { problems }\end{array}$ & $\begin{array}{l}\text { Social } \\
\text { support }\end{array}$ & \\
\hline \multirow[t]{2}{*}{58} & \multirow{2}{*}{$\begin{array}{l}\text { Glazier RH., } \\
\text { et al., } \\
\text { Canada, } \\
2004 \text { [112] }\end{array}$} & \multirow{2}{*}{$\begin{array}{l}\text { Emotional/ } \\
\text { instrumen- } \\
\text { tal support }\end{array}$} & \multirow[t]{2}{*}{$\begin{array}{l}\text { Family/ } \\
\text { friends/ } \\
\text { partner }\end{array}$} & \multirow[t]{2}{*}{2052} & \multirow[t]{2}{*}{ Facility } & Longitudinal & $\begin{array}{l}\text { Depression: } \\
\text { CESD, }\end{array}$ & \multirow[t]{2}{*}{ MSPSS } & $r=-7.38, P<0.01$ \\
\hline & & & & & & Longitudinal & Anxiety: STAl & & $r=-7.34, P<0.01$ \\
\hline \multirow[t]{3}{*}{59} & \multirow{3}{*}{$\begin{array}{l}\text { Senturk V., } \\
\text { et al., } 2011 \\
\text { [113] }\end{array}$} & $\begin{array}{l}\text { General sup- } \\
\text { port }\end{array}$ & Partner & \multirow[t]{3}{*}{772} & \multirow[t]{3}{*}{ Facility } & \multirow[t]{3}{*}{$\begin{array}{l}\text { Cross-sec- } \\
\text { tional }\end{array}$} & \multirow[t]{3}{*}{$\begin{array}{l}\text { Depression: } \\
\text { EPDS }\end{array}$} & \multirow[t]{3}{*}{$\mathrm{CPQ}$} & $\beta=-2.6(-3.6,-1.7)$ \\
\hline & & $\begin{array}{c}\text { Emotional } \\
\text { support }\end{array}$ & $\begin{array}{l}\text { Mother in- } \\
\text { low }\end{array}$ & & & & & & $\beta=-2.6,95 \% \mathrm{Cl}(-4.6,-1.9)$ \\
\hline & & $\begin{array}{l}\text { Practical } \\
\text { support }\end{array}$ & & & & & & & $\beta=-0.8,95 \% \mathrm{Cl}(-1.4,-0.3)$ \\
\hline \multirow[t]{2}{*}{60} & \multirow{2}{*}{$\begin{array}{l}\text { Gausia k., } \\
\text { et al., } \\
\text { Bangla- } \\
\text { desh, } 2009 \\
\text { [18] }\end{array}$} & \multirow[t]{2}{*}{$\begin{array}{l}\text { General sup- } \\
\text { port }\end{array}$} & $\begin{array}{l}\text { Mother in- } \\
\text { low }\end{array}$ & \multirow[t]{2}{*}{361} & \multirow[t]{2}{*}{ Community } & \multirow[t]{2}{*}{$\begin{array}{l}\text { Cross-sec- } \\
\text { tional }\end{array}$} & \multirow[t]{2}{*}{$\begin{array}{l}\text { Depression: } \\
\text { EPDS }\end{array}$} & \multirow[t]{2}{*}{ PPP } & AOR:2.41(1.31, 4.45) \\
\hline & & & Partner & & & & & & AOR: $8.26(1.66,41)$ \\
\hline 61 & $\begin{array}{l}\text { Shidhaye P., } \\
\text { et al., India, } \\
2017 \text { [114] }\end{array}$ & $\begin{array}{l}\text { General sup- } \\
\text { port }\end{array}$ & $\begin{array}{l}\text { Friend/ } \\
\text { partner/ } \\
\text { relatives }\end{array}$ & 302 & Facility & $\begin{array}{l}\text { Cross-sec- } \\
\text { tional }\end{array}$ & $\begin{array}{l}\text { Depression: } \\
\text { EPDS }\end{array}$ & PSS & AOR: $3.33(1.42,5)$ \\
\hline 62 & $\begin{array}{l}\text { Hartley } \\
\text { M., et al., } \\
\text { South } \\
\text { Africa, } \\
2011 \text { [115] }\end{array}$ & $\begin{array}{c}\text { Emotional/ } \\
\text { practical } \\
\text { support }\end{array}$ & $\begin{array}{l}\text { Family/part- } \\
\text { ner }\end{array}$ & 1062 & Facility & Longitudinal & $\begin{array}{l}\text { Depression: } \\
\text { EPDS }\end{array}$ & CPQ & AOR: $1.13(1.03,1.25)$ \\
\hline 63 & $\begin{array}{l}\text { Rwakarema } \\
\text { M et al., } \\
\text { Tanzania, } \\
2017 \text { [116] }\end{array}$ & $\begin{array}{l}\text { General sup- } \\
\text { port }\end{array}$ & $\begin{array}{l}\text { Family/ } \\
\text { friends/ } \\
\text { partner }\end{array}$ & 397 & Facility & $\begin{array}{l}\text { Cross-sec- } \\
\text { tional }\end{array}$ & $\begin{array}{l}\text { Depression: } \\
\text { EPDS }\end{array}$ & MSSS & AOR: $1.41(0.60,3.28)$ \\
\hline 64 & $\begin{array}{l}\text { Heyningen } \\
\text { Tet al., } \\
\text { South } \\
\text { Africa, } \\
2016 \text { [117] }\end{array}$ & $\begin{array}{l}\text { Emotional/ } \\
\text { instrumen- } \\
\text { tal support }\end{array}$ & $\begin{array}{l}\text { Family/ } \\
\text { friends/ } \\
\text { partner }\end{array}$ & 376 & Facility & $\begin{array}{l}\text { Cross-sec- } \\
\text { tional }\end{array}$ & $\begin{array}{l}\text { Depression: } \\
\text { EPDS }\end{array}$ & MSPSS & AOR: $1.14(1.06,1.22)$ \\
\hline 65 & $\begin{array}{l}\text { e Couto T., } \\
\text { et al., Brazil, } \\
2016 \text { [43] }\end{array}$ & $\begin{array}{l}\text { General sup- } \\
\text { port }\end{array}$ & $\begin{array}{l}\text { Partner/ } \\
\text { parents/ } \\
\text { parents-in- } \\
\text { law }\end{array}$ & 255 & Facility & $\begin{array}{l}\text { Cross-sec- } \\
\text { tional }\end{array}$ & $\begin{array}{l}\text { Self-harm: } \\
\text { MINI }\end{array}$ & OSSS-3 & AOR: $1.75(0.62,5)$ \\
\hline 66 & $\begin{array}{l}\text { Pinheiro RT., } \\
\text { et al., Brazil, } \\
2012 \text { [118] }\end{array}$ & $\begin{array}{l}\text { Emotional/ } \\
\text { instrumen- } \\
\text { tal support }\end{array}$ & $\begin{array}{l}\text { Family/ } \\
\text { friends/ } \\
\text { partner }\end{array}$ & 871 & Facility & $\begin{array}{l}\text { Cross-sec- } \\
\text { tional }\end{array}$ & $\begin{array}{l}\text { Self-harm: } \\
\text { MINI }\end{array}$ & $\begin{array}{l}\text { MOS-SSS } \\
\text { (7 item) }\end{array}$ & AOR: $3.03(1.78,5.26)$ \\
\hline 67 & $\begin{array}{l}\text { Supraja, TA., } \\
\text { et al., } 2016 \\
\text { India [119] }\end{array}$ & $\begin{array}{l}\text { General sup- } \\
\text { port }\end{array}$ & $\begin{array}{l}\text { Spouse, } \\
\text { other fam- } \\
\text { ily mem- } \\
\text { bers, and } \\
\text { friends }\end{array}$ & 462 & Facility & $\begin{array}{l}\text { Cross-sec- } \\
\text { tional }\end{array}$ & $\begin{array}{l}\text { Self-harm: } \\
\text { SBQ-R }\end{array}$ & MSSS-8 item & AOR: $1.17(1.02,2.35)$ \\
\hline
\end{tabular}

being good quality, which provides insights into the robustness of our meta-analysis (Additional file 3).

\section{Narrative analysis}

\section{Association between social support and antenatal depression}

Fifteen studies that investigated the association [72, 82, $99,101,104,110]$ or correlation $[59,79,81,93,106,112]$ between social support and antenatal depression were included in the narrative analysis. Among these 15 studies, 6 report a significant negative correlation between social support and antenatal depression [59, 79, 81, 93, 106, 112]. Also, a significant inverse relationship between social support and antenatal depression was reported by 8 studies [39, 72, 82, 99, 101, 104, 110, 114] and one study (conducted in Jordan) [59] reported no evidence of a significant correlation between social support and antenatal depression. 


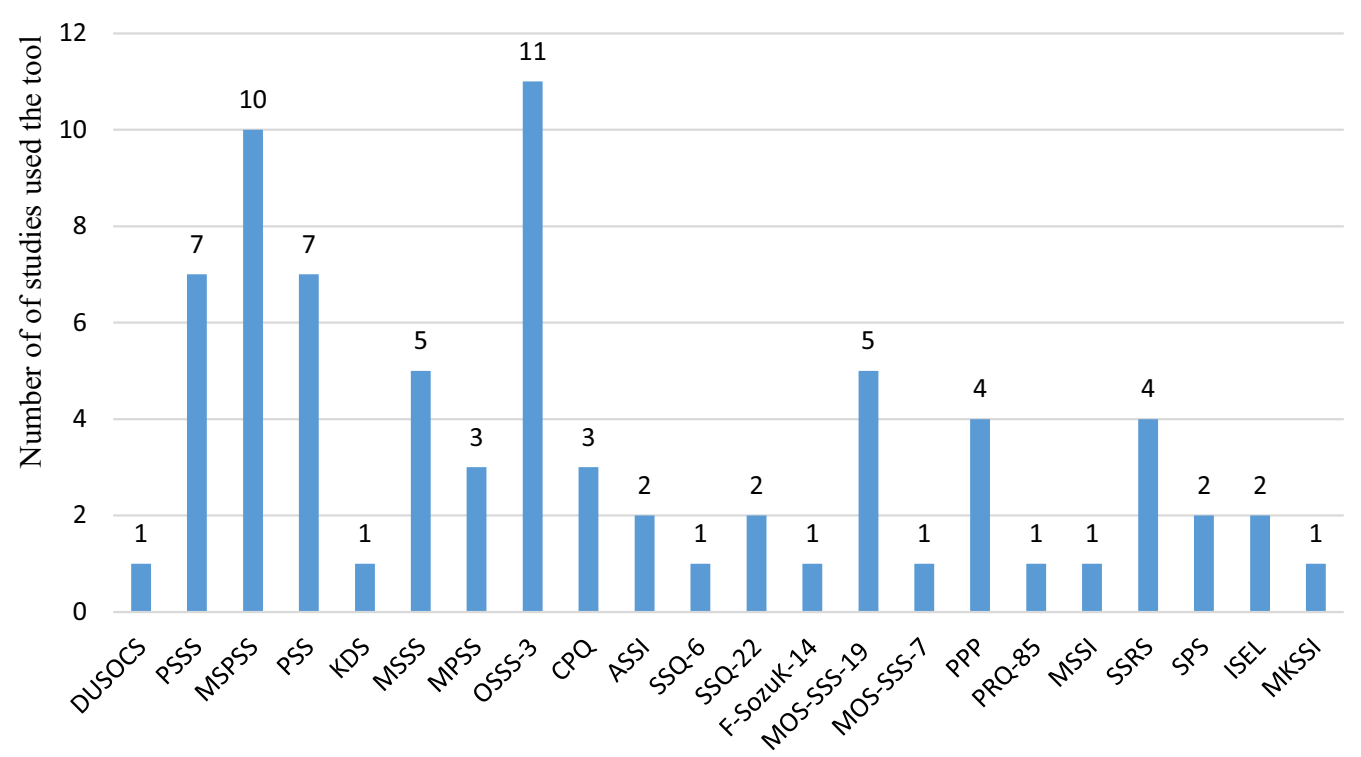

Type of social support tool

Fig. 2 Types of social support tools used by the studies included in the current study

Among four studies conducted in the US, an inverse relation between social support and antenatal depression was reported from a longitudinal facility-based study conducted on 106 pregnant mothers, during the first trimester $(\beta=-0.44, \mathrm{P}<0.001)$ and second trimester $(\beta=-0.33, P<0.001)$ [72]. Similarly, another facility-based cross-sectional study reported negative $(\beta$ : $-0.751, P<0.001)[82]$, and positive association $(\beta=1.64, \mathrm{P}<0.001)$ between social support and antenatal depression [104]. The fourth study which recruited pregnant women through a stratified random sampling technique revealed a negative correlation $(\mathrm{n}=325, \mathrm{r}=-0.25$, $\mathrm{P}<0.001)$ between social support and antenatal depression [112].

Also, among three studies conducted in Turkey, negative moderate correlation between the EPDS score and perceived social support was reported from a facility-based cross-sectional study $(n=258, r=-0.43$; $\mathrm{P}<0.001)$ [79]. Another facility-based cross-sectional study reported that social support was significantly related to depression $(\mathrm{n}=655, \beta=2.421,95 \%$ CI $(0.707$, 4.135) [101]. In addition, another facility-based study indicated that support from husband $(\mathrm{n}=772, \beta=-2.6$ ( $-3.6,-1.7)$, emotional support $(\beta=-2.6,95 \% \mathrm{CI}$ $(-4.6,-1.9)$ and practical support $(\beta=-0.8,95 \% \mathrm{CI}$ $(-1.4,-0.3))$ from mother in low has inverse relation with antenatal depression [114].

Negative correlation between social support and antenatal depression was reported from longitudinal studies conducted in Germany $(\mathrm{n}=297, \mathrm{r}=-0.45, \mathrm{p}<0.01)$
[81] and Canada $(\mathrm{n}=2052, \mathrm{r}=-7.38, \mathrm{P}<0.01)$ [113]. Similarly, a negative correlation was reported from facility-based cross-sectional studies conducted in Iran $(\mathrm{r}=-0.642, \mathrm{p}<0.001)$ [93] and Pakistan $(\mathrm{r}=-0.453$, $\mathrm{P}<0.001)$ [106]. A cross-sectional study conducted in Canada reported that social support was negatively associated with antenatal depression $(n=364, \beta:-0.32$, $\mathrm{P}<0.001)$ [99].

A study conducted in Australia on consecutively selected pregnant mothers emphasized that good social support was negatively associated with depression during pregnancy $(\mathrm{n}=367, \beta=-0.18, \mathrm{P}<0.001)$ [39]. Similarly, a study from Taiwan reported an inverse relationship between social support and antenatal depression $(\mathrm{n}=158, \beta=-0.44,95 \%$ CI $(-0.63,-0.24), \mathrm{P}<0.05)$ [110]. Despite the above evidence of association, a crosssectional study conducted in Jordan concluded that social support during pregnancy has no correlation with antenatal depression $(\mathrm{r}=-0.022, \mathrm{P}>0.05)$ [59].

\section{Association between social support and antenatal anxiety}

Eight studies examined the association between social support and anxiety during pregnancy. Of which seven studies reported significant association $[80,108,110]$ or correlation $[62,93,106,113]$ between social support and antenatal anxiety. However, one study conducted in Canada [77] reported no evidence of a significant association between social support and antenatal anxiety.

A longitudinal study conducted in the United Kingdom has shown that women receiving greater social support 
Table 2 Social support tools used, concepts measured and their reliability

\begin{tabular}{|c|c|c|c|}
\hline S. no. & Type of Social support tool used & Concepts measured & Cronbach Alpha \\
\hline 1 & $\begin{array}{l}\text { The Duke Social Support and Stress Scale (DUSOCS) (12 } \\
\text { items) [119] }\end{array}$ & $\begin{array}{l}\text { Family and non-family relationships in terms of the amount } \\
\text { of social support they provide and number of supportive } \\
\text { people }\end{array}$ & 0.74 \\
\hline 2 & $\begin{array}{l}\text { Multidimensional Scale of Perceived Social Support (MSPSS) } \\
\text { (12 items) [120] }\end{array}$ & $\begin{array}{l}\text { The subjective adequacy of emotional and instrumental } \\
\text { social support from three different sources (family, friends, } \\
\text { and partner) }\end{array}$ & 0.83 \\
\hline 3 & Kuesioner Dukungan Sosial (KDS) (24 item) [121] & $\begin{array}{l}\text { Support from husband, extended families from both sides, } \\
\text { environmental support, mother's preparedness, and } \\
\text { traditional rituals }\end{array}$ & 0.788 \\
\hline 4 & $\begin{array}{l}\text { Medical Outcomes Study Social Support Survey (MOS-SSS) } \\
\text { (7 items) [35] }\end{array}$ & $\begin{array}{l}\text { Perceived emotional and instrumental support from one's } \\
\text { social network }\end{array}$ & 0.88 \\
\hline 5 & $\begin{array}{l}\text { Medical Outcomes Study Social Support Survey (MOS-SSS) } \\
\text { (19 items) [35] }\end{array}$ & $\begin{array}{l}\text { Perceived emotional/informational support, tangible sup- } \\
\text { port, affectionate support, and positive social interaction }\end{array}$ & 0.97 \\
\hline 6 & Oslo Social Support Scale(OSSS-3 item) [122] & Support received from husband, parents, and parents-in-law & 0.88 \\
\hline 7 & Close Person Questionnaire (CPQ) [123] & $\begin{array}{l}\text { Emotional support, practical support, and negative aspects } \\
\text { of the relationship }\end{array}$ & 0.85 \\
\hline 8 & Partner social support Scale (PSSS) [124] & $\begin{array}{l}\text { Support received from a partner (emotional, instrumental } \\
\text { and being dependent on partner) }\end{array}$ & 0.89 \\
\hline 9 & Prenatal Psychosocial Profile (PPP) [125] & Social support from partner and others & 0.71 \\
\hline 10 & Arizona Social Support Interview (ASSI) [126] & $\begin{array}{l}\text { Availability of support from social network/family: instru- } \\
\text { mental, emotional, informative, normative, and compan- } \\
\text { ionship }\end{array}$ & 0.7 \\
\hline 11 & Maternal Social Support Index (MSSI) (18 items) [127] & $\begin{array}{l}\text { Support received from family, friend, and her feeling about } \\
\text { the support. Also, the support received on routine home } \\
\text { duties (watching children, doing other activities) }\end{array}$ & 0.81 \\
\hline 12 & $\begin{array}{l}\text { Modified Kendler Social support interview (MKSSI) (27 items) } \\
\text { [128] }\end{array}$ & Emotional and instrumental support & 0.68 \\
\hline 13 & Social Support rating scale (SSRS) (10 items) [129] & $\begin{array}{l}\text { Objective support, subjective support, and support seeking } \\
\text { behavior }\end{array}$ & 0.76 \\
\hline 14 & Social provision Scale (SPS) (24 items) [130] & $\begin{array}{l}\text { Intimacy, social integration, a reassurance of worth, and sup- } \\
\text { port from friends, family members, coworkers, community } \\
\text { members, and so on }\end{array}$ & 0.81 \\
\hline 15 & Social support Questionnaire (12 items) [131] & $\begin{array}{l}\text { Emotional support, instrumental support, social integration } \\
\text { and measure perceptions of social support and satisfaction } \\
\text { with that social support }\end{array}$ & 0.81 \\
\hline 16 & Social support Questionnaire-6 (SSQ-6 item) [132] & $\begin{array}{l}\text { Availability of social support/number of supporters (SSQ- } \\
\text { Network) and satisfaction with social support (SSQ- } \\
\text { Satisfaction) }\end{array}$ & 0.83 \\
\hline 17 & $\begin{array}{l}\text { F-SozU K-14 (Fragebogen zur Sozialen Unterst€utzung; } \\
\text { Social Support Questionnaire) (14 items) [133] }\end{array}$ & $\begin{array}{l}\text { The perceived or anticipated emotional and instrumental } \\
\text { support from one's social environment }\end{array}$ & 0.94 \\
\hline 18 & Interpersonal support Evaluation list (ISEL) (40 items) [134] & $\begin{array}{l}\text { Perceived availability of aspects of social support like emo- } \\
\text { tional, belonging, tangible, and self-esteem }\end{array}$ & 0.74 \\
\hline 19 & Personal resource questionnaire (PRQ-85) (85 items) [135] & $\begin{array}{l}\text { Assessment of the number of resources a person can count } \\
\text { across life situations and a person's satisfaction with these } \\
\text { resources }\end{array}$ & 0.84 \\
\hline 20 & Perceived Social Support (PSS) (25 items) [136] & Social support from partner, friend, relatives, and co-worker & 0.75 \\
\hline 21 & Social relationship Scale (SRS) [137] & Perceived availability of emotional and material support & 0.87 \\
\hline 22 & Maternal social Support Scale (MSSS) (6 items) [138] & Level of perceived support from family, friends, and husband & 0.74 \\
\hline
\end{tabular}

from their family reported feeling significantly less anxious; one standard deviation (SD) increase in social support (SS) from the family is associated with a 0.044 SD decrease in anxiety $(\mathrm{P}=0.029)$. Also, a one SD increase in social support from the husband/partner was associated with a 0.033 SD decrease in STAI-State scores $(\mathrm{P}=0.0051)[62]$.

Another finding from a longitudinal study in Bangladesh $(\mathrm{n}=720)(\beta:-1.144, \mathrm{P}<0.05)[108]$ and Taiwan $(\mathrm{n}=158,(\beta=-0.79,95 \%$ CI $(-1.16,-0.42), \mathrm{P}<0.05)$ [110] reported that social support during pregnancy was 
negatively associated with anxiety. A cross-sectional study conducted in Iran $(\mathrm{n}=372)(\mathrm{r}=-0.456, \mathrm{p}<0.001)$ [93] and Pakistan $(\mathrm{n}=500, \mathrm{r}=-0.433, \mathrm{P}<0.001)$ [106] among pregnant women revealed that there was a significant negative correlation between social support and anxiety during pregnancy. Similarly, a negative correlation was also reported from a longitudinal study conducted in Canada ( $\mathrm{n}=2052, \mathrm{r}=-7.34, \mathrm{P}<0.01)$ [113].

A facility-based study conducted in Greece on pregnant mothers concluded that there was no significant correlation between good social support and antenatal anxiety elation $(n=165, \beta=0.131,95 \%$ CI $(0.19,2.37), P>0.05)$ [80]. A facility-based longitudinal study conducted in Canada reported social support did not have significant relation with antenatal anxiety $(\mathrm{n}=5271, \beta=0.08,95 \%$ CI $(0.01,0.15), \mathrm{P}>0.05)$ [77].

\section{Association between social support and self-harm during pregnancy}

Due to the small number of studies examining selfharm and low social support among pregnant women, no meta-analysis was conducted on this specific association, thereby included in the narrative analysis. Three cross-sectional studies examined the association between social supports and self-harm during pregnancy. A crosssectional study conducted in South Africa among randomly selected pregnant women reported a significant association between social support and suicidal ideation and behaviour (SIB) during pregnancy $(n=376$, AOR: $1.07,95 \%$ CI $(1.01,1.15), \mathrm{P}<0.05)$, suggesting a protective effect of good social support [89]. A cross-sectional study conducted in Brazil, which employed a consecutive sampling process to recruit pregnant women, reported that women with low social support were 3 times more likely to develop self-harm compared with their counterparts ( $\mathrm{n}=871$, AOR: 3.03 , 95\% CI $(1.78,5.26)$ [119]. Finally, a study conducted in India among urban pregnant women, found that those who reported low perceived social support had a higher odds of developing current suicidal ideation ( $\mathrm{n}=462$, AOR: 1.17, 95\% CI $(1.02,2.35)$. However, a cross-sectional study, conducted in Brazil among 255 pregnant mothers, found no significant association between social support and self-harm (AOR: 1.75, 95\% CI $(0.62,5)[43]$.

\section{Meta-analysis of the association between low social support and antenatal depression}

Drawing upon data from 45 studies identified, a metaanalysis was conducted to examine the association between low social support and antenatal depression. From these 45 studies, 36 (80\%) were conducted at the health facility level and 26 (57\%) employed a cross-sectional study design. Also, 29 (64\%) of the studies used the Edinburgh Postnatal Depression Scale (EPDS) as a screening tool to measure depression. From the identified 45 studies, a relatively large number of papers (20 [44.4\%]) were published between the year 2016-2019 (Table 1).

Except for eight studies [68, 74, 87, 90, 94, 103, 111, 117] all the remaining 37 studies included in the metaanalysis revealed low social support has a significant positive association with the risk of antenatal depression. The result of the meta-analysis showed low social support has a significant positive association with antenatal depression (AOR: 2.00 (95\% CI: 1.71, 2.34) (Fig. 3). As the eggers test was found significant $(p=0.033)$, the final pooled effect size was corrected using Duval and Tweedie's trim and fill analysis (AOR: 1.18 (95\% CI: $1.01,1.41)$. Due to the observed significant heterogeneity $\left(\mathrm{I}^{2}=98.9 \%, \mathrm{Q}=3962.35, \mathrm{df}=44, \mathrm{P}<0.001\right)$ a random effect meta-analysis model was employed. To identify the possible sources of heterogeneity, variables such as publication year (Coefficient: - 0.019, P: 0.301) and sample size (Coefficient: - 0.0001, P: 0.019) were investigated via a univariate meta-regression model, and the sample size was statistically significant and identified as one of the possible sources of heterogeneity.

\section{Subgroup analysis of the association between low social support and antenatal depression}

Due to the reported high heterogeneity index among studies examining the association between low social support and antenatal depression, a subgroup analysis was conducted using characteristics like study setting, the income of countries, study design, sample size, publication year and tools used.

The subgroup analysis conducted based on the study setting revealed a higher POR of low social support among studies conducted at community setting $\left(\mathrm{POR}=2.21,95 \% \mathrm{CI}: 1.25,3.93, \mathrm{I}^{2}=99 \%, \mathrm{Q}=792.08\right.$, $\mathrm{P}<0.001) \quad$ compared with facility-based studies $\left(\mathrm{POR}=2.21,95 \%\right.$ CI $(1.25,3.93), \mathrm{I}^{2=} 93.0 \%, \mathrm{Q}=502.3$, $\mathrm{P}<0.001)$. In addition, a higher POR was estimated in the years 2000-2005, $(\mathrm{POR}=4.37,95 \%$ CI $(2.20,8.71$, $\left.\mathrm{I}^{2}=36.0 \%, \mathrm{P}<0.001\right)$ followed by the years 2006-2010 $\left(\mathrm{POR}=2.20,95 \% \mathrm{CI}(1.31,3.71), \mathrm{I}^{2}=88.1 \%, \mathrm{P}<0.001\right)$. Regarding the median sample size, the POR of low social support was relatively higher among studies with a sample size greater than $520(\mathrm{POR}=2.01,95 \% \mathrm{CI}(1.59,2.55)$, $\mathrm{I}^{2}=88.9 \%, \mathrm{P}<0.001$ ) (Table 3 ).

\section{Sensitivity analysis}

A leave-one-out sensitivity analysis was conducted among studies examining the association between low social support and antenatal depression to help identify 


\section{Study
ID}

ES $(95 \%$ Cl $) \quad$ Weight

Adewuya AO., et al (2007)

Agostini F., et al (2015)

Anindyajati G., et al (2017)

Bayrampour H., et al (2015)

Belay YB., et al (2018)

Bernard O., et al (2018)

Biratu A., et al (2015)

Bisetegn TA., et al (2016)

Cankorur vs., et al (2015)

Chee C., et al (2005)

Cheng E., et al (2016)

Dibaba Y., et al (2013)

Dong X., et al (2013)

Dong X., et al (2013)

Dong X., et al (2013)

Dudas R., at al (2012)

Duko B., et al (2019)

Fall A., et al (2013)

Gao L., et al (2019)

Jeong H., et al (2013)

Lau Y., et al (2011)

Lee AM., et al (2007)

Li Y., et al (2017)

Pajulo M., et al (2001)

Rashid A., et al (2017)

Rubertsson C.et al (2002)

Sheeba B., et al (2019)

Sidebottom AC., et al (2017)

Spoozak L., et al (2008)

Stewart RC., et al (2014)

Xian T., et al (2019)

Woldetensay YK., et al (2018)

Zeng Y., et al (2015)

Westdahl C.,et al (2008)

Sahile MA., et al (2017)

Nasreen HE ., et al (2011)

Martini J. et al (2015)

Rubertsson C.,et al (2010)

Jesse ED., et 1 (2005)

Gausia k., et al (2009)

Gausia k., et al (2009)

Shidhaye P., et al (2017)

Hartley M.,et al (2011)

Rwakarema M et al (2015)

Heyningen $\mathrm{T}$ et al (2015)

Overall (I-squared $=98.9 \%, \mathrm{p}=0.000)$

NOTE: Weights are from random effects analysis

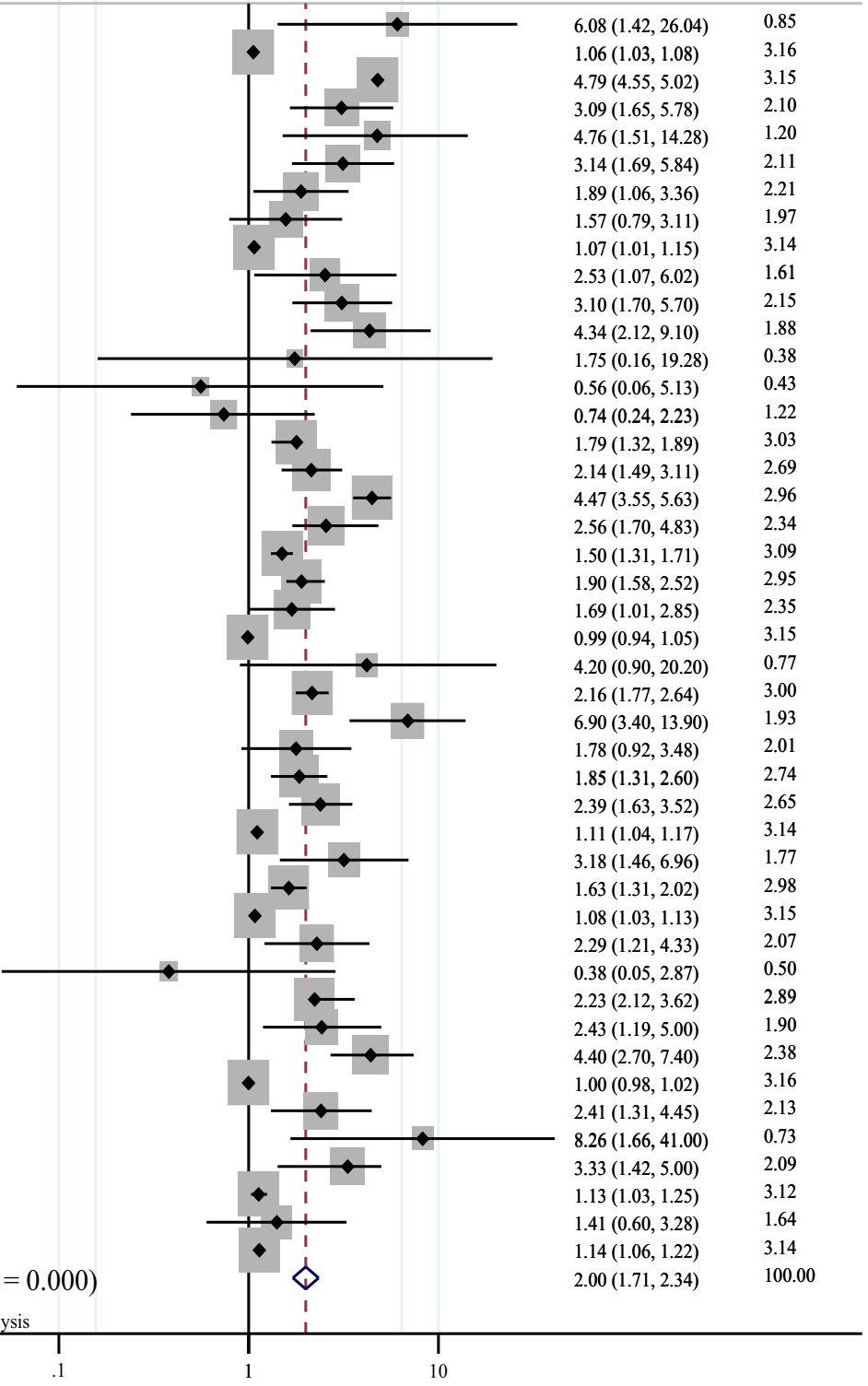

Fig. 3 Forest plot indicating POR of low social support as a risk factor for antenatal depression

the effect of a single study on the overall pooled estimate. The sensitivity analysis using the random-effects model resulted in the POR ranges from 1.94 (95\% CI: 1.66, 2.28) to 2.07 (95\% CI: $1.71,2.49)$. The sensitivity analysis shows that none of these studies was found to have substantially altered the overall results of the analysis.

\section{Meta-analysis of the association between low social} support and antenatal anxiety

A meta-analysis was also conducted drawing upon data reported from 9 papers which examined the association between low social support and antenatal anxiety. From these studies, 8 were institution based cross-sectional studies and $6(66.6 \%)$ used longitudinal study design and 5 reported data from high-income countries.

All the studies included in this meta-analysis found that low social support has a significant association with the 
Table 3 Subgroup analysis of odds ratios of low social support in the association between social support and antenatal depression $(\mathrm{N}=45,2000-2019)$, (random effect model)

\begin{tabular}{|c|c|c|c|c|}
\hline \multirow[t]{2}{*}{ Variable } & \multirow[t]{2}{*}{ No. of studies } & \multirow[t]{2}{*}{$\begin{array}{l}\text { Pooled AOR } \\
(95 \% \mathrm{Cl})\end{array}$} & \multicolumn{2}{|c|}{$\begin{array}{l}\text { Heterogeneity within the study }\left(I^{2} \text { and }\right. \\
Q)\end{array}$} \\
\hline & & & $\mathrm{Q}$ value & $I^{2}, P$-value \\
\hline \multicolumn{5}{|l|}{ Study setting } \\
\hline Facility & 36 & $1.63(1.49,1.77)$ & 502.3 & $93.0 \%, p<0.001$ \\
\hline Community & 9 & $2.21(1.25,3.93)$ & 792.0 & $99.0 \%, p<0.001$ \\
\hline \multicolumn{5}{|l|}{ Income of country } \\
\hline High-income & 16 & $1.98(1.72,2.27)$ & 367 & $95.9 \%, p<0.001$ \\
\hline Middle-income & 14 & $1.33(1.19,1.50)$ & 115.7 & $88.8 \%, p<0.001$ \\
\hline Low-income & 15 & $2.26(1.36,3.76)$ & 1431.5 & $99.0 \%, p<0.001$ \\
\hline \multicolumn{5}{|l|}{ Study design } \\
\hline Cross-sectional study & 26 & $1.84(1.4,2.41)$ & 3534.2 & $99.3 \%, p<0.001$ \\
\hline Longitudinal study & 19 & $2.1(1.78,2.48)$ & 339.6 & $94.7 \%, p<0.001$ \\
\hline \multicolumn{5}{|l|}{ Median sample size $e^{a}$} \\
\hline$<520$ & 30 & $1.99(1.63,2.41)$ & 3833.8 & $99.2 \%, p<0.001$ \\
\hline$>=520$ & 15 & $2.02(1.59,2.55)$ & 126.5 & $88.9 \%, p<0.001$ \\
\hline \multicolumn{5}{|l|}{ Publication year } \\
\hline 2000-2005 & 3 & $4.37(2.20,8.71)$ & 3.13 & $36.0 \%, p=0.209$ \\
\hline 2006-2010 & 7 & $2.20(1.31,3.71)$ & 50.3 & $88.1 \%, p<0.001$ \\
\hline $2011-2015$ & 15 & $1.67(1.43,1.95)$ & 295.8 & $95.3 \%, p<0.001$ \\
\hline 2016-2019 & 20 & $2.06(1.43,2.97)$ & 2598.4 & $99.3 \%, p<0.001$ \\
\hline \multicolumn{5}{|c|}{ Depression assessment tool } \\
\hline Screening & 41 & $2.07(1.74,2.46)$ & 3940.9 & $99.0 \%, p<0.001$ \\
\hline Diagnostic & 4 & $1.29(1.09,1.52)$ & 19.25 & $84.4 \%, p<0.001$ \\
\hline
\end{tabular}

${ }^{\mathrm{a}}$ Cut of point is based on the median of sample size $($ median $=520)$

risk of antenatal anxiety $[64,71,78,83,86,88,98,105$, 109]. The pooled estimate found that low social support has a significant positive association with antenatal anxiety (AOR: 2.28 (95\% CI: 1.47, 3.54) (Fig. 4). As the eggers test was found significant $(\mathrm{p}<0.001)$, the final pooled effect size was corrected using Duval and Tweedie's trim and fill analysis (AOR: 1.97 (95\% CI: 1.34, 2.92). Since we found significant heterogeneity among the studies $\left(\mathrm{I}^{2}=90.0 \% \%, \mathrm{Q}=79.82, \mathrm{df}=8, \mathrm{P}<0.001\right)$ a random effect meta-analysis model was applied. To identify the possible sources of heterogeneity, variables such as publication year (Coefficient: 0.02, P: 0.688) and sample size (Coefficient: 0.0002, P: 0.261) were investigated using univariate meta-regression models, but none of these variables was found to be statistically significant.

\section{Subgroup analysis of the association between low social support and antenatal anxiety}

Due to the reported high heterogeneity index among studies examining the association between low social support and antenatal anxiety studies, a subgroup analysis was conducted using characteristics like study setting, the income of countries, study design, sample size, publication year and tools used.

The sub-group analysis undertaken based on the design of the study revealed a higher POR of low social support was among studies conducted using a longitudinal study design (POR: 2.59, 95\% CI (1.87, 3.57), $\left.\mathrm{I}^{2}=44.2 \%, \mathrm{Q}=8.97, \mathrm{P}=0.11\right)$. In addition, a subgroup meta-analysis conducted based on the income of countries reported that higher POR of low social support was among high-income countries determined a POR of 2.34 (95\% CI $(1.76,3.11), \mathrm{I}^{2}=23.6 \%, \mathrm{Q}=5.23$, $\mathrm{p}=0.264$ ) (Table 4).

\section{Publication bias}

With regards to the literature reporting on the association of low social support with antenatal depression and antenatal anxiety, a funnel plot for both meta-analyses appeared asymmetrical indicating the presence of publication bias and Egger's test for antenatal depression $(\mathrm{P}=0.033)$ and antenatal anxiety $(\mathrm{P}<0.001)$ also showed evidence of publication bias. In response, Duval and Tweedie's trim and fill analysis was conducted. 


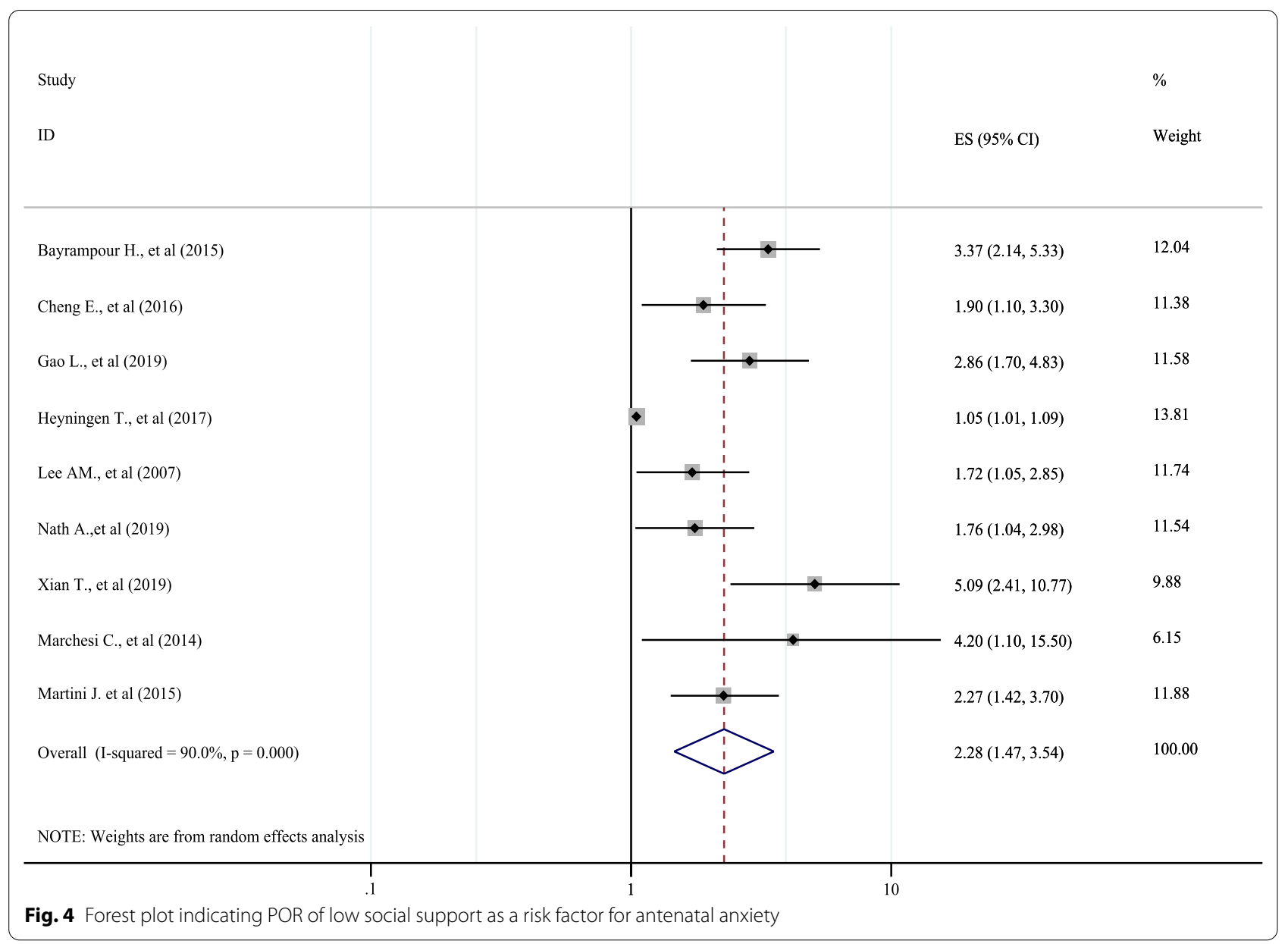

Table 4 Subgroup analysis of odds ratios of association between low social support and antenatal anxiety ( $N=9,2000-2019)$ (random effect model)

\begin{tabular}{|c|c|c|c|c|}
\hline \multirow[t]{2}{*}{ Variable } & \multirow[t]{2}{*}{ No. of studies } & \multirow[t]{2}{*}{$\begin{array}{l}\text { Pooled AOR } \\
(95 \% \mathrm{Cl})\end{array}$} & \multicolumn{2}{|c|}{$\begin{array}{l}\text { Heterogeneity within the study } \\
\left(I^{2} \text { and } Q\right)\end{array}$} \\
\hline & & & $Q$ value & $I^{2}, P$-value \\
\hline \multicolumn{5}{|l|}{ Income of country } \\
\hline High-income & 5 & $2.34(1.76,3.11)$ & 5.23 & $23.6 \%, p=0.264$ \\
\hline Low and middle-income & 4 & $2.15(1.06,4.38)$ & 34.55 & $91.3 \%, p<0.001$ \\
\hline \multicolumn{5}{|l|}{ Study design } \\
\hline Cross-sectional study & 3 & $2.27(1.46,3.53)$ & 17.68 & $88.7 \%, p<0.001$ \\
\hline Longitudinal study & 6 & $2.59(1.87,3.57)$ & 8.97 & $44.2 \%, P=0.11$ \\
\hline \multicolumn{5}{|l|}{ Median sample size $e^{a}$} \\
\hline$<376$ & 4 & $2.28(1.72,3.02)$ & 2.77 & $0 \%, P=0.429$ \\
\hline$>=376$ & 5 & $2.17(1.16,4.07)$ & 49.53 & $91.9 \%, p<0.001$ \\
\hline \multicolumn{5}{|l|}{ Publication year } \\
\hline $2015+$ & 7 & $2.27(1.36,3.78)$ & 72.45 & $91.7 \%, P<0.001$ \\
\hline Before 2015 & 2 & $2.16(1.007,4.63)$ & 1.53 & $34.7 \%, P=0.216$ \\
\hline
\end{tabular}

${ }^{\mathrm{a}}$ Cut of point is based on the Median of sample size (median $\left.=376\right)$ 


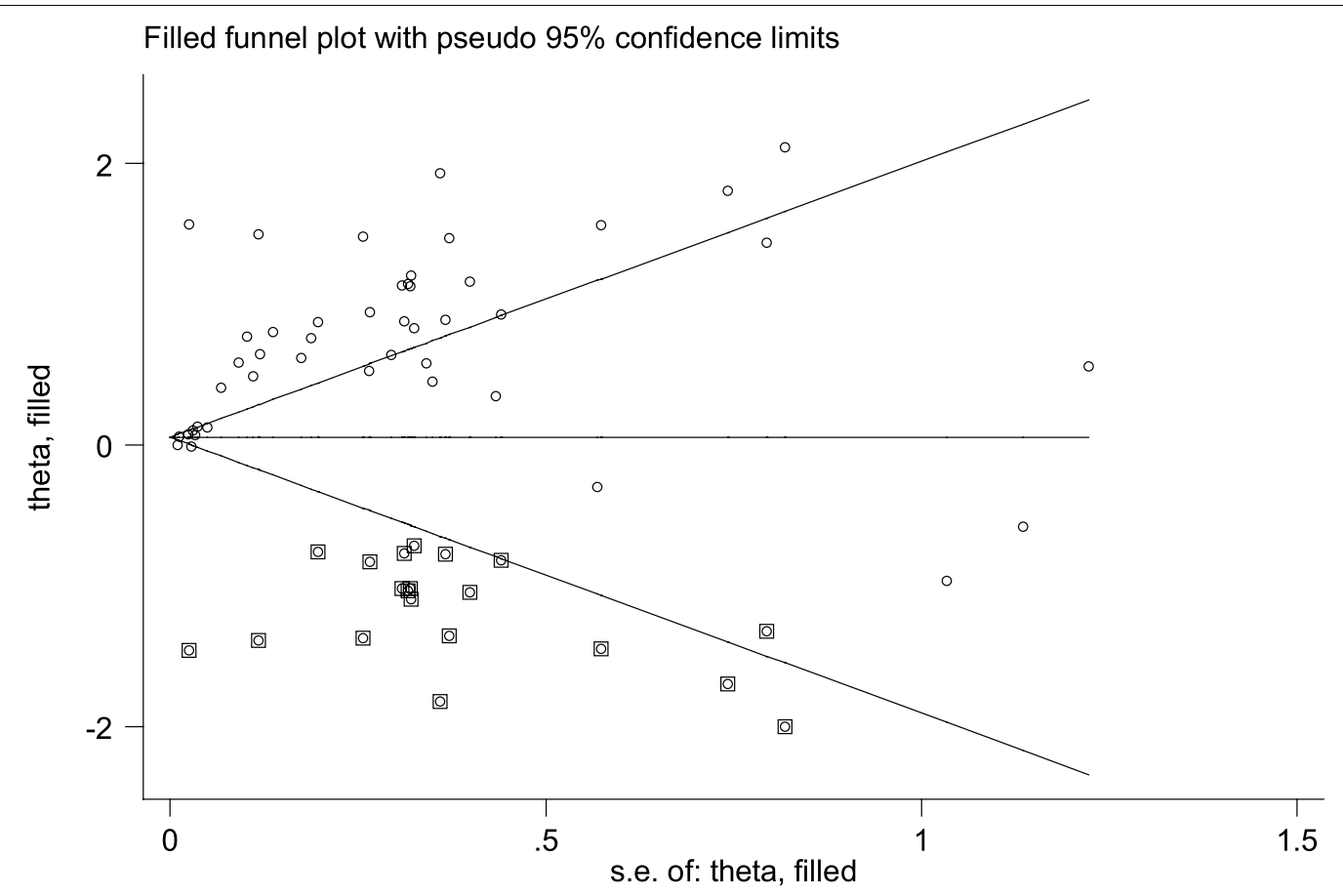

Fig. 5 Tweedie's and Duval's trim and fill analysis on studies examining the association between low social support and antenatal depression

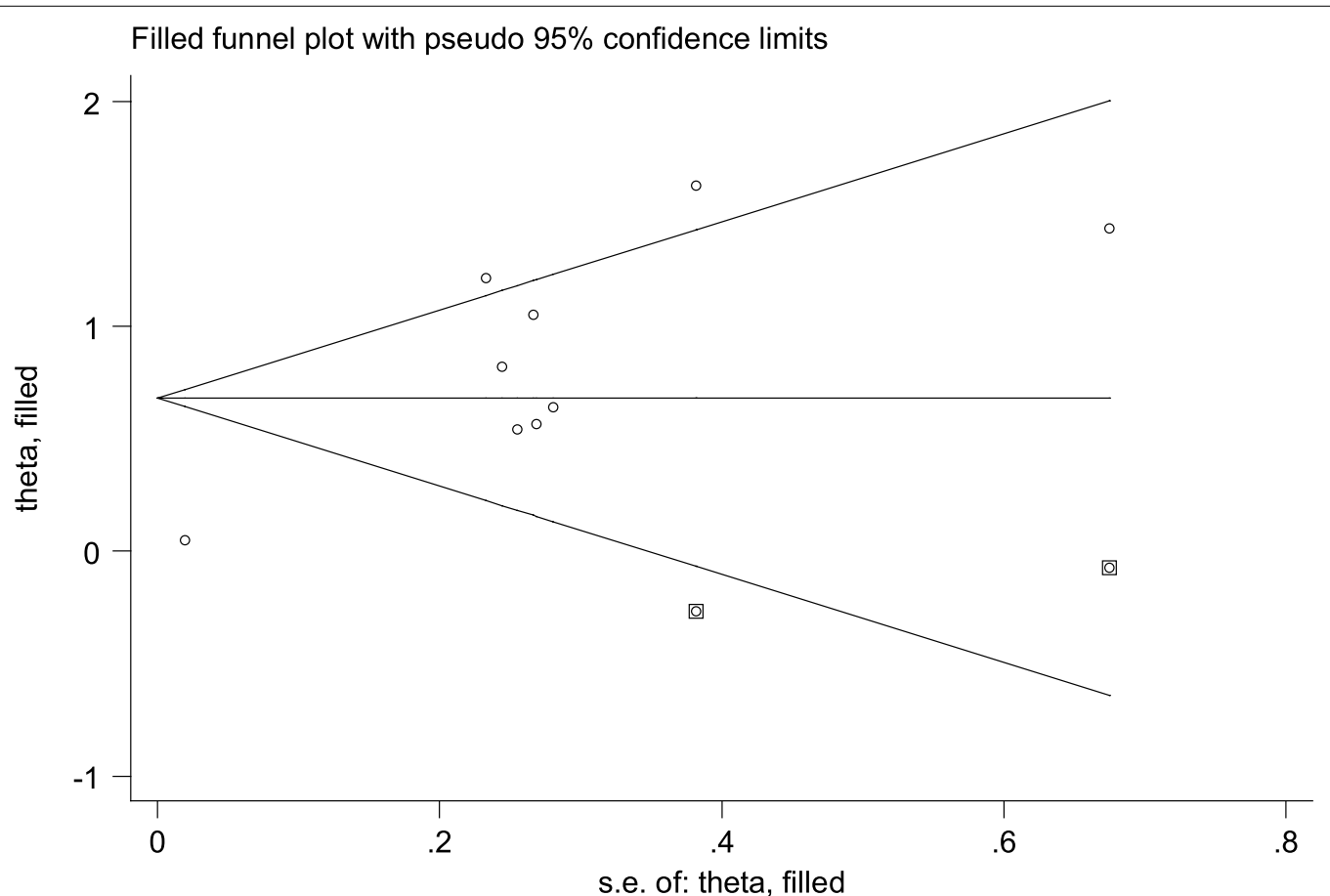

Fig. 6 Tweedie's and Duval's trim and fill analysis on studies examining the association between low social support and antenatal anxiety

After adjusting for the publication bias, the trim and fill analysis reported an estimate of pooled AOR of low social support reduced from AOR: 2.00 (95\% CI: 1.47,
3.54) to AOR: 1.18 (95\% CI: 1.01, 1.41) for antenatal depression (Fig. 5) and from AOR: 2.28 (95\% CI: 1.47, 
3.54) to AOR: 1.97 (95\% CI: 1.34, 2.92) for antenatal anxiety (Fig. 6).

\section{Discussion}

Our study reports the findings of the first systematic review and meta-analysis examining the relationship between social support and mental illness (depression, anxiety, and self-harm) during pregnancy, revealing a number of interesting findings.

Our review identified that pregnant women who received low social support are more likely to develop mental illness compared to pregnant women who received good social support. Among studies included in the narrative synthesis, a majority of studies reported significant positive associations between low social support and antenatal depression [14/15 (93.3\%) studies], low social support and antenatal anxiety [6/8 (75\%) studies] and low social support and self-harm [3/4 (75\%) studies] during pregnancy. Further, the pooled estimate of the meta-analysis shows that low social support had a significant positive association with antenatal depression and antenatal anxiety. Pregnant women with low social support may not have someone to confide in, obtain important information/advice from, or help reduce the negative emotions associated with a distressing situation, and as a result, they might be exposed to stress and may later develop depression [139]. Also, pregnant women with low social support are less satisfied with family and poor in interacting with the social environment, and as a result, they might be exposed to loneliness, become less in emotional and stress coping ability and later become more anxious [139, 140].

Support for our findings comes from various epidemiological studies conducted in general populations that have revealed low social support was associated with the onset and relapse of depression among individuals with a previous history of mental illness [141], and seasonal change of mood disorder in UK [142]. Also, low social support has a significant positive relationship with postnatal depression among a representative sample of Australian women [143].

A global level systematic review found that social support is also associated with improved mental health and decreased levels of depressive symptoms among female heads of households [144]. Good social support [145] may play a protective role against mental illness during pregnancy. Pregnant women who have good social support are more likely to have improved mental, psychological, and emotional health compared with their counterparts [6, 37]. Also, another research finding showed that individuals with constructive social relations and good social support enjoy more efficient communication skills, helping provide some protection from depression and other mental illnesses [146]. On the other hand, good social support protects people from illnesses [147] and can help provide an additional coping mechanism for stress [145].

A randomized control trial (RCT) examining the psychosocial benefits of a telephone support program for pregnant women in the metropolitan city on the South Island of New Zeeland revealed the intervention group at 34 weeks had lower stress scores, lower trait anxiety and less depressed mood than the control group [148]. However, another randomized controlled trial conducted in North East England involving low risk nulliparous pregnant women found that provision of additional telephone support by a midwife did not significantly reduce anxiety level $(p=0.68)$ [149]. Similarly, another RCT conducted in the US among pregnant women with a history of at least one spontaneous perinatal loss, found that providing intervention like home visits and support by nurses found no significant decrease in anxiety scores between the groups post-intervention $(\mathrm{p}=0.66)$ [150].

As presented in sub-group analyses, among studies examining the association between low social support and antenatal depression, the pooled odds ratio of low social support was relatively higher among studies conducted in low-income countries compared with studies conducted in high-income countries. This might be due to the fact that most women living in low-income and middle-income countries face financial and economic challenges which might expose them to additional stress and social exclusion compared with pregnant women living in high-income countries [151]. Also, involvement in social activities may require money to attend events. So social exclusion and self-isolation of individuals from the social environment might lead them to feelings of loneliness and other psychological problems [152]. This concept was supported by a study conducted in Germany, which identified that socially disadvantaged persons more often report poor social networks and social support compared with their counterpart [153].

Finally, there was a significant level of heterogeneity amongst the studies examining the association between social support and either antenatal anxiety or antenatal depression. This high level of heterogeneity could be due to the different conceptualisation and measurement of social support employed in the studies. Our review identified, 22 different types of social support assessment tools used to measure social support. This shows a difference in the understanding of social support across many individuals and community members who were from different countries with different socio-economic settings. The lack of comprehensive agreement regarding the best method to measure social support is one of the identified challenge across the current literature [36]. As a result, 
work towards a unified social support measurement would be helpful.

\section{Implications for future research and clinical practice}

Our review found that low social support has significant associations with the risk of mental health problems (depression, anxiety, and self-harm) during pregnancy. This suggests maternal health professionals need to have discussions with pregnant women regarding their level and source of social support. Policymakers and other relevant stakeholders should consider helping develop community-based social support programs for pregnant women to effectively integrate alongside other commonly used maternal health services. Reverse causation is possible between low social support and mental health problems during pregnancy. Therefore, to address the issue of reverse causation, future longitudinal studies, which can ensure the temporal order of events, is recommended. Finally, future interventional research is needed to further explore the effect of social support in preventing mental health problems during pregnancy.

\section{Limitations}

There were some limitations to our study. The search was restricted to only include studies published in English language, which may lead to publication bias. Due to variations in diagnostic approaches, the assessments used for social support, depression and anxiety may be prone to measurement bias. However, we have addressed the issue of heterogeneity and publication bias during our analysis, which provides better estimates of the associations between social support and depression and anxiety during pregnancy.

\section{Conclusion}

Low social support has significant associations with depression, anxiety, and self-harm during pregnancy. Strong social support may act to safeguard pregnant women from depression, anxiety, and self-harm. Maternal health professionals need to have discussions with pregnant women regarding the level and source of social support they receive and to also monitoring pregnant women's mental health status if she is considered to have low social support. Maternal health professionals may also wish to consider encouraging the social network of pregnant women to improve social support being given. Policymakers and other relevant stakeholders should consider helping develop community-based social support programs for pregnant women that can be effectively integrated with other commonly used maternal health services. Finally, future research in this area should consider interventional studies that explore the effectiveness of social support in preventing mental illness during pregnancy.

\section{Abbreviations}

ASSI: Arizona social support interview; BAI: Beck anxiety inventory; BDI: Beck's depression inventory tool; BIPS: Behaviour in pregnancy study tool; CESD: Centre for Epidemiologic Studies Depression Scale; Cl: Confidence interval; CIDI: Composite international diagnostic intervention; CPQ: Close person questionnaire; DUSOCS: The Duke Social Support and Stress Scale; EPDS: Edinburgh Postnatal Depression Scale; HAMA: Hamilton Anxiety Scale (HAMA); ISEL: Interpersonal support evaluation list; MINI: Mini International Neuropsychiatric Interview diagnostic interview; MKSSI: Modified Kendler Social Support Interview; MOSS: The Medical Outcomes Study Social Support Scale; MSOS-SSS: The 19-item Medical Outcomes Study Social Support Survey; MSPSS: Multidimensional Scale of Perceived Social Support; MSSI: Maternal Social Support Index; MSSS: Maternity Social Support Scale; NOS: Newcastle-Ottawa Scale; OSSS-3: 3 Items Oslo Social Support Scale; SBQ-R: Revised Suicidal Behaviour Questionnaire.

\section{Supplementary Information}

The online version contains supplementary material available at https://doi. org/10.1186/s12978-021-01209-5.

Additional file 1. PRISMA 2020 Checklist.

Additional file 2. Data extraction sheet used for studies examining the relationship between social support and mental health problems (depression, anxiety and self-harm) among adult pregnant mothers.

Additional file 3. Newcastle Ottawa (NOS) critical appraisal evaluation for Cross sectional and Cohort studies.

\section{Acknowledgements}

No acknowledgements at this stage.

\section{Authors' contributions}

The author $A B$ performed the search, quality appraisal, data extraction, analyses, and draft of the initial manuscript. DS and JA participated in consensus. DS, WP, and JA contributed to the analysis, quality appraisal, revising the draft manuscript. All authors read and approved the final manuscript.

Funding

The authors declare that there is no funding.

Availability of data and materials

All data generated or analyzed data during this review are included in this article and its additional file.

\section{Declarations}

Ethics approval and consent to participate Not applicable.

\section{Consent for publication}

Not applicable.

\section{Competing interests}

The authors declare that there is no competing interest.

\section{Author details}

${ }^{1}$ College of Medicine and Health Sciences, School of Nursing, Hawassa University, Hawassa, Ethiopia. ${ }^{2}$ Australian Centre for Public and Population Health Research, School of Public Health, Faculty of Health, University of Technology Sydney, Ultimo, NSW, Australia. 
Received: 18 October 2020 Accepted: 19 July 2021

Published online: 28 July 2021

\section{References}

1. Davalos DB, Yadon CA, Tregellas HC. Untreated prenatal maternal depression and the potential risks to offspring: a review. Arch Womens Ment Health. 2012:15:1-14.

2. Smith MV, Shao L, Howell H, Lin H, Yonkers KA. Perinatal depression and birth outcomes in a Healthy Start project. Matern Child Health J. 2011;15:401-9.

3. Diego MA, Jones NA, Field T, Hernandez-Reif M, Schanberg S, Kuhn C, Gonzalez-Garcia A. Maternal psychological distress, prenatal cortisol, and fetal weight. Psychosom Med. 2006;68:747-53.

4. Brown SJ, Yelland JS, Sutherland GA, Baghurst PA, Robinson JS. Stressful life events, social health issues and low birth weight in an Australian population-based birth cohort: challenges and opportunities in antenatal care. BMC Public Health. 2011;11:196.

5. Divney AA, Sipsma H, Gordon D, Niccolai L, Magriples U, Kershaw T. Depression during pregnancy among young couples: the effect of personal and partner experiences of stressors and the buffering effects of social relationships. J Pediatr Adolesc Gynecol. 2012;25:201-7.

6. Dunkel Schetter C. Psychological science on pregnancy: stress processes, biopsychosocial models, and emerging research issues. Annu Rev Psychol. 2011;62:531-58.

7. Rich-Edwards JW, Kleinman K, Abrams A, Harlow BL, McLaughlin TJ, Joffe H, Gillman MW. Sociodemographic predictors of antenatal and postpartum depressive symptoms among women in a medical group practice. J Epidemiol Commun Health. 2006;60:221-7.

8. Say L, Chou D, Gemmill A, Tunçalp Ö, Moller A-B, Daniels J, Gülmezoglu AM, Temmerman M, Alkema L. Global causes of maternal death: a WHO systematic analysis. Lancet Glob Health. 2014;2:e323-33.

9. Huizink AC, Mulder EJ, de Medina PGR, Visser GH, Buitelaar JK. Is pregnancy anxiety a distinctive syndrome? Early Hum Dev. 2004;79:81-91.

10. Rico MAG, Rodríguez AJM, Díez SMU, Real MCM. Análisis de la relación entre riesgo gestacional y ansiedad materna. Prog Obstet Ginecol. 2010;53:273-9.

11. Hernández-Martínez C, Val VA, Murphy M, Busquets PC, Sans JC. Relation between positive and negative maternal emotional states and obstetrical outcomes. Women Health. 2011;51:124-35.

12. Nieminen K, Stephansson O, Ryding EL. Women's fear of childbirth and preference for cesarean section - a cross-sectional study at various stages of pregnancy in Sweden. Acta Obstet Gynecol Scand. 2009:88:807-13.

13. Teixeira C, Figueiredo B, Conde A, Pacheco A, Costa R. Anxiety and depression during pregnancy in women and men. J Affect Disord. 2009:119:142-8.

14. Dennis C-L, Falah-Hassani K, Shiri R. Prevalence of antenatal and postnatal anxiety: systematic review and meta-analysis. Br J Psychiatry. 2017:210:315-23

15. Lopez AD, Murray CJ. The global burden of disease: a comprehensive assessment of mortality and disability from diseases, injuries, and risk factors in 1990 and projected to 2020; summary. Harvard School of Public Health; 1996.

16. Kastrup MC, Ramos AB. Global mental health. Dan Med Bull. 2007:54:42-3

17. Dadi AF, Miller ER, Bisetegn TA, Mwanri L. Global burden of antenatal depression and its association with adverse birth outcomes: an umbrella review. BMC Public Health. 2020;20:173.

18. Gausia K, Fisher C, Ali M, Oosthuizen J. Antenatal depression and suicidal ideation among rural Bangladeshi women: a community-based study. Arch Womens Ment Health. 2009;12:351-8.

19. Frautschi S, Cerulli A, Maine D. Suicide during pregnancy and its neglect as a component of maternal mortality. Int I Gynecol Obstet. 1994;47:275-84

20. Newport DJ, Levey L, Pennell P, Ragan K, Stowe ZN. Suicidal ideation in pregnancy: assessment and clinical implications. Arch Womens Ment Health. 2007;10:181-7.

21. Lindahl V, Pearson $\mathrm{JL}$, Colpe L. Prevalence of suicidality during pregnancy and the postpartum. Arch Womens Ment Health. 2005;8:77-87.
22. Orr ST, Miller CA. Maternal depressive symptoms and the risk of poor pregnancy outcome: review of the literature and preliminary findings. Epidemiol Rev. 1995:17:165-71.

23. Kurki T, Hiilesmaa V, Raitasalo R, Mattila H, Ylikorkala O. Depression and anxiety in early pregnancy and risk for preeclampsia. Obstet Gynecol. 2000;95:487-90

24. Freeman MP. Perinatal psychiatry: risk factors, treatment data, and specific challenges for clinical researchers. J Clin Psychiatry. 2008;69:633-4.

25. Steer RA, Scholl TO, Hediger ML, Fischer RL. Self-reported depression and negative pregnancy outcomes. J Clin Epidemiol. 1992;45:1093-9.

26. Hollins K. Consequences of antenatal mental health problems for child health and development. Curr Opin Obstet Gynecol. 2007;19:568-72.

27. Stott D. Follow-up study from birth of the effects of prenatal stresses. Dev Med Child Neurol. 1973;15:770-87.

28. Heron J, O'Connor TG, Evans J, Golding J, Glover V, Team AS. The course of anxiety and depression through pregnancy and the postpartum in a community sample. J Affect Disord. 2004;80:65-73.

29. Figueiredo B, Costa R, Pacheco A, Pais A. Mother-to-infant emotional involvement at birth. Matern Child Health J. 2009;13:539-49.

30. Hart S, Field T, del Valle C, Pelaez-Nogueras M. Depressed mothers' interactions with their one-year-old infants. Infant Behav Dev. 1998;21:519-25.

31. Straub H, Qadir S, Miller G, Borders A. Stress and stress reduction. Clin Obstet Gynecol. 2014;57:579-606.

32. Shapiro GD, Fraser WD, Frasch MG, Séguin JR. Psychosocial stress in pregnancy and preterm birth: associations and mechanisms. J Perinat Med. 2013:41:631-45.

33. Orr ST, Miller CA. Unintended pregnancy and the psychosocial wellbeing of pregnant women. Womens Health Issues. 1997;7:38-46.

34. Norbeck JS, DeJoseph JF, Smith RT. A randomized trial of an empiricallyderived social support intervention to prevent low birthweight among African American women. Soc Sci Med. 1996:43:947-54.

35. Sherbourne CD, Stewart AL. The MOS social support survey. Soc Sci Med. 1991;32:705-14

36. Berkman LF. Social support, social networks, social cohesion and health Soc Work Health Care. 2000;31:3-14.

37. Cohen S, Wills TA. Stress, social support, and the buffering hypothesis. Psychol Bull. 1985;98:310.

38. Edwards B, Galletly C, Semmler-Booth T, Dekker G. Antenatal psychosocial risk factors and depression among women living in socioeconomically disadvantaged suburbs in Adelaide, South Australia. Aust N Z J Psychiatry. 2008:42:45-50

39. Leigh B, Milgrom J. Risk factors for antenatal depression, postnatal depression and parenting stress. BMC Psychiatry. 2008:8:24.

40. Ogbo FA, Eastwood J, Hendry A, Jalaludin B, Agho KE, Barnett B, Page A. Determinants of antenatal depression and postnatal depression in Australia. BMC Psychiatry. 2018;18:49.

41. Biaggi A, Conroy S, Pawlby S, Pariante CM. Identifying the women at risk of antenatal anxiety and depression: a systematic review. J Affect Disord. 2016;191:62-77.

42. Nasreen HE, Kabir ZN, Forsell Y, Edhborg M. Prevalence and associated factors of depressive and anxiety symptoms during pregnancy: a population based study in rural Bangladesh. BMC Womens Health. 2011;11:1-9.

43. e Couto TC, Brancaglion MYM, Cardoso MN, Faria GC, Garcia FD, Nicolato R, Aguiar RALP, Leite HV, Corrêa H. Suicidality among pregnant women in Brazil: prevalence and risk factors. Arch Womens Ment Health. 2016:19:343-8.

44. Page MJ, McKenzie JE, Bossuyt PM, Boutron I, Hoffmann TC, Mulrow CD, Shamseer L, Tetzlaff JM, Moher D. Updating guidance for reporting systematic reviews: development of the PRISMA 2020 statement. J Clin Epidemiol. 2021;134:103-12.

45. Kellermeyer L, Harnke B, Knight S. Covidence and rayyan. J Med Libr Assoc. 2018:106:580.

46. Cowan N. The magical number 4 in short-term memory: a reconsideration of mental storage capacity. Behav Brain Sci. 2001:24:87-114.

47. APA: American Psychiatric Association. Diagnostic and statistical manual of mental disorder version 5. 2012, 4

48. Cohen MM, Ansara D, Schei B, Stuckless N, Stewart DE. Posttraumatic stress disorder after pregnancy, labor, and delivery. J Women's Health (15409996). 2004:13:315-24. 
49. Stang A. Critical evaluation of the Newcastle-Ottawa scale for the assessment of the quality of nonrandomized studies in meta-analyses. Eur J Epidemiol. 2010;25:603-5.

50. Moher D, Shamseer L, Clarke M, Ghersi D, Liberati A, Petticrew M, Shekelle P, Stewart LA. Preferred reporting items for systematic review and meta-analysis protocols (PRISMA-P) 2015 statement. Syst Rev. 2015. https://doi.org/10.1186/2046-4053-4-1.

51. Borenstein M, Hedges LV, Higgins JP, Rothstein HR. A basic introduction to fixed-effect and random-effects models for meta-analysis. Res Synth Methods. 2010;1:97-111.

52. Higgins JP, Thompson SG, Deeks JJ, Altman DG. Measuring inconsistency in meta-analyses. BMJ Br Med J. 2003;327:557-60.

53. Egger M, Smith GD, Schneider M, Minder C. Bias in meta-analysis detected by a simple, graphical test. BMJ. 1997;315:629-34.

54. Ioannidis JP. Interpretation of tests of heterogeneity and bias in metaanalysis. J Eval Clin Pract. 2008;14:951-7.

55. Aitkin M. A general maximum likelihood analysis of variance components in generalized linear models. Biometrics. 1999;55:117-28.

56. Higgins JP, Thompson SG. Quantifying heterogeneity in a meta-analysis. Stat Med. 2002;21:1539-58.

57. Herbell K, Zauszniewski JA. Stress experiences and mental health of pregnant women: the mediating role of social support. Issues Mental Health Nursing. 2019;40:613-20

58. Fall A, Goulet L, Vézina M. Comparative study of major depressive symptoms among pregnant women by employment status. Springerplus. 2013:2:1-11.

59. Abujilban SK, Abuidhail J, Al-Modallal H, Hamaideh S, Mosemli O. Predictors of antenatal depression among Jordanian pregnant women in their third trimester. Health Care Women Int. 2014;35:200-15.

60. Adewuya AO, Ola BA, Aloba OO, Dada AO, Fasoto OO. Prevalence and correlates of depression in late pregnancy among Nigerian women. Depress Anxiety. 2007;24:15-21.

61. Agostini F, Neri E, Salvatori P, Dellabartola S, Bozicevic L, Monti F. Antenatal depressive symptoms associated with specific life events and sources of social support among Italian women. Matern Child Health J. 2015;19:1131-41.

62. Akiki S, Avison WR, Speechley KN, Campbell MK. Determinants of maternal antenatal state-anxiety in mid-pregnancy: Role of maternal feelings about the pregnancy. J Affect Disord. 2016;196:260-7.

63. Anindyajati G, Ismail RI, Diatri H, Elvira SD. Antenatal depression and its determinant factors in urban community setting. Adv Sci Lett. 2017;23:3439-41.

64. Bayrampour H, McDonald S, Tough S. Risk factors of transient and persistent anxiety during pregnancy. Midwifery. 2015;31:582-9.

65. Belay YA, Moges NA, Hiksa FF, Arado KK, Liben ML. Prevalence of antenatal depression and associated factors among pregnant women attending antenatal care at Dubti Hospital: a case of pastoralist region in Northeast Ethiopia. Depress Res Treat. 2018;2018:1659089.

66. Bernard O, Gibson RC, McCaw-Binns A, Reece J, Coore-Desai C, Shakespeare-Pellington S, Samms-Vaughan M. Antenatal depressive symptoms in Jamaica associated with limited perceived partner and other social support: a cross-sectional study. PLoS ONE. 2018;13: e0194338.

67. Biratu A, Haile D. Prevalence of antenatal depression and associated factors among pregnant women in Addis Ababa, Ethiopia: a crosssectional study. Reprod Health. 2015;12:99.

68. Bisetegn TA, Mihretie G, Muche T. Prevalence and predictors of depression among pregnant women in Debretabor, Town Northwest Ethiopia. PLOS ONE. 2016;11: e0161108.

69. Cankorur VS, Abas M, Berksun O, Stewart R. Social support and the incidence and persistence of depression between antenatal and postnatal examinations in Turkey: a cohort study. BMJ Open. 2015;5: e006456.

70. Chee CY, Lee DT, Chong Y, Tan L, Ng T, Fones CS. Confinement and other psychosocial factors in perinatal depression: a transcultural study in Singapore. J Affect Disord. 2005;89:157-66.

71. Cheng ER, Rifas-Shiman SL, Perkins ME, Rich-Edwards JW, Gillman MW Wright $R$, Taveras EM. The influence of antenatal partner support on pregnancy outcomes. J Womens Health (Larchmt). 2016;25:672-9.

72. Clements AD, Fletcher TR, Childress LD, Montgomery RA, Bailey BA. Social support, religious commitment, and depressive symptoms in pregnant and postpartum women. J Reprod Infant Psychol. 2016;34:247-59.

73. Dibaba Y, Fantahun M, Hindin MJ. The association of unwanted pregnancy and social support with depressive symptoms in pregnancy: evidence from rural Southwestern Ethiopia. BMC Pregnancy Childbirth. 2013;13:135.

74. Dong X, Qu Z, Liu F, Jiang X, Wang Y, Chui CH, Wang X, Tian D, Zhang $X$. Depression and its risk factors among pregnant women in 2008 Sichuan earthquake area and non-earthquake struck area in China. J Affect Disord. 2013;151:566-72.

75. Dudas RB, Csatordai S, Devosa I, Toreki A, Ando B, Barabas K, Pal A, Kozinszky Z. Obstetric and psychosocial risk factors for depressive symptoms during pregnancy. Psychiatry Res. 2012;200:323-8.

76. Duko B, Ayano G, Bedaso A. Depression among pregnant women and associated factors in Hawassa city, Ethiopia: an institution-based crosssectional study. Reprod Health. 2019;16:25.

77. Dunkel Schetter C, Niles AN, Guardino CM, Khaled M, Kramer MS. Demographic, medical, and psychosocial predictors of pregnancy anxiety. Paediatr Perinat Epidemiol. 2016;30:421-9.

78. Gao L, Qu J, Wang AY. Anxiety, depression and social support in pregnant women with a history of recurrent miscarriage: a cross-sectional study. J Reproduct Infant Psychol. 2020.38:497-508.

79. Golbasi Z, Kelleci M, Kisacik G, Cetin A. Prevalence and correlates of depression in pregnancy among Turkish women. Matern Child Health J. 2010;14:485-91.

80. Gourounti K, Anagnostopoulos F, Lykeridou K. Coping strategies as psychological risk factor for antenatal anxiety, worries, and depression among Greek women. Arch Womens Ment Health. 2013;16:353-61.

81. Hain S, Oddo-Sommerfeld S, Bahlmann F, Louwen F, SchermellehEngel K. Risk and protective factors for antepartum and postpartum depression: a prospective study. J Psychosom Obstet Gynaecol. 2016;37:119-29.

82. Herbell K, Zauszniewski JA. Stress experiences and mental health of pregnant women: the mediating role of social support. Issues Ment Health Nurs. 2019:40:613-20.

83. van Heyningen T, Honikman S, Myer L, Onah MN, Field S, Tomlinson M. Prevalence and predictors of anxiety disorders amongst low-income pregnant women in urban South Africa: a cross-sectional study. Arch Womens Ment Health. 2017;20:765-75.

84. Jeong H-G, Lim J-S, Lee M-S, Kim S-H, Jung I-K, Joe S-H. The association of psychosocial factors and obstetric history with depression in pregnant women: focus on the role of emotional support. Gen Hosp Psychiatry. 2013;35:354-8.

85. Lau Y. A longitudinal study of family conflicts, social support, and antenatal depressive symptoms among Chinese women. Arch Psychiatr Nurs. 2011:25:206-19.

86. Lee AM, Lam SK, Lau SMSM, Chong CSY, Chui HW, Fong DYT. Prevalence, course, and risk factors for antenatal anxiety and depression. Obstet Gynecol. 2007;110:1102-12.

87. Li Y, Long Z, Cao D, Cao F. Social support and depression across the perinatal period: a longitudinal study. J Clin Nurs. 2017;26:2776-83.

88. Nath A, Venkatesh S, Balan S, Metgud CS, Krishna M, Murthy GVS. The prevalence and determinants of pregnancy-related anxiety amongst pregnant women at less than 24 weeks of pregnancy in Bangalore Southern India. Int J Womens Health. 2019;11:241-8.

89. Onah MN, Field S, Bantjes J, Honikman S. Perinatal suicidal ideation and behaviour: psychiatry and adversity. Arch Womens Ment Health. 2017;20:321-31.

90. Pajulo M, Savonlahti E, Sourander A, Helenius H, Piha J. Antenatal depression, substance dependency and social support. J Affect Disord. 2001;65:9-17.

91. Rashid A, Mohd R. Poor social support as a risk factor for antenatal depressive symptoms among women attending public antennal clinics in Penang, Malaysia. Reprod Health. 2017;14:144.

92. Rubertsson C, Waldenström U, Wickberg B. Depressive mood in early pregnancy: prevalence and women at risk in a national Swedish sample. J Reprod Infant Psychol. 2003;21:113-23.

93. Shafaie FS, Mirghafourvand M, Rahmati M, Nouri P, Bagherinia M. Association between psychological status with perceived social support in pregnant women referring to Tabriz health centers. J Matern Fetal Neonatal Med. 2018;31:1554-60. 
94. Sheeba B, Nath A, Metgud CS, Krishna M, Venkatesh S, Vindhya J, Murthy GVS. Prenatal depression and its associated risk factors among pregnant women in Bangalore: a hospital based prevalence study. Front Public Health. 2019;7:108.

95. Sidebottom AC, Hellerstedt WL, Harrison PA, Jones-Webb RJ. Prenatal care: associations with prenatal depressive symptoms and social support in low-income urban women. Arch Womens Ment Health. 2017;20:633-44.

96. Spoozak L, Gotman N, Smith MV, Belanger K, Yonkers KA. Evaluation of a social support measure that may indicate risk of depression during pregnancy. J Affect Disord. 2009;114:216-23.

97. Stewart RC, Umar E, Tomenson B, Creed F. A cross-sectional study of antenatal depression and associated factors in Malawi. Arch Womens Ment Health. 2014;17:145-54.

98. Tang X, Lu Z, Hu D, Zhong X. Influencing factors for prenatal Stress, anxiety and depression in early pregnancy among women in Chongqing, China. J Affect Disord. 2019;253:292-302.

99. Verreault N, Da Costa D, Marchand A, Ireland K, Dritsa M, Khalife S. Rates and risk factors associated with depressive symptoms during pregnancy and with postpartum onset. J Psychosom Obstet Gynaecol. 2014;35:84-91.

100. Woldetensay YK, Belachew T, Biesalski HK, Ghosh S, Lacruz ME, Scherbaum V, Kantelhardt EJ. The role of nutrition, intimate partner violence and social support in prenatal depressive symptoms in rural Ethiopia: community based birth cohort study. BMC Pregnancy Childbirth. 2018;18:374

101. Yanikkerem E, Ay S, Mutlu S, Goker A. Antenatal depression: prevalence and risk factors in a hospital based Turkish sample. J Pak Med Assoc. 2013;63:472-7.

102. Zeng Y, Cui Y, Li J. Prevalence and predictors of antenatal depressive symptoms among Chinese women in their third trimester: a crosssectional survey. BMC Psychiatry. 2015;15:66.

103. Sahile MA, Segni MT, Awoke T, Bekele D. Prevalence and predictors of antenatal depressive symptoms among women attending Adama Hospital Antenatal Clinic, Adama, Ethiopia. Int J Nurs Midwifery. 2017:9:58-64

104. Records K, Rice M. Psychosocial correlates of depression symptoms during the third trimester of pregnancy. J Obstet Gynecol Neonatal Nurs. 2007;36:231-42.

105. Marchesi C, Ampollini P, Paraggio C, Giaracuni G, Ossola P, De Panfilis C, Tonna M, Viviani D. Risk factors for panic disorder in pregnancy: a cohort study. J Affect Disord. 2014:156:134-8.

106. Waqas A, Raza N, Lodhi HW, Muhammad Z, Jamal M, Rehman A. Psychosocial factors of antenatal anxiety and depression in Pakistan: is social support a mediator? PLOS ONE. 2015;10: e0116510.

107. Westdahl C, Milan S, Magriples U, Kershaw TS, Rising SS, Ickovics JR. Social support and social conflict as predictors of prenatal depression. Obstet Gynecol. 2007;1 10:134-40.

108. Nasreen HE, Kabir ZN, Forsell Y, Edhborg M. Prevalence and associated factors of depressive and anxiety symptoms during pregnancy: a population based study in rural Bangladesh. BMC Womens Health. 2011;11:22.

109. Martini J, Petzoldt J, Einsle F, Beesdo-Baum K, Hofler M, Wittchen HU. Risk factors and course patterns of anxiety and depressive disorders during pregnancy and after delivery: a prospective-longitudinal study. J Affect Disord. 2015;175:385-95.

110. Huang MZ, Kao CH, Lin KC, Hwang JL, Puthussery S, Gau ML. Psychological health of women who have conceived using assisted reproductive technology in Taiwan: findings from a longitudinal study. BMC Womens Health. 2019;19:97.

111. Jesse DE, Walcott-McQuigg J, Mariella A, Swanson MS. Risks and protective factors associated with symptoms of depression in low-income African American and Caucasian women during pregnancy. J Midwifery Womens Health. 2005;50:405-10.

112. Blaney NT, Fernandez MI, Ethier KA, Wilson TE, Walter E, Koenig LJ. Psychosocial and behavioral correlates of depression among HIV-infected pregnant women. AIDS Patient Care STDS. 2004;18:405-15.

113. Glazier R, Elgar F, Goel V, Holzapfel S. Stress, social support, and emotional distress in a community sample of pregnant women. J Psychosom Obstet Gynaecol. 2004;25:247-55.
114. Senturk V, Abas M, Berksun O, Stewart R. Social support and antenatal depression in extended and nuclear family environments in Turkey: a cross-sectional survey. BMC Psychiatry. 2011;11:48.

115. Shidhaye P, Shidhaye R, Phalke V. Association of gender disadvantage factors and gender preference with antenatal depression in women: a cross-sectional study from rural Maharashtra. Soc Psychiatry Psychiatr Epidemiol. 2017:52:737-48.

116. Hartley M, Tomlinson M, Greco E, Comulada WS, Stewart J, le Roux I, Mbewu N, Rotheram-Borus MJ. Depressed mood in pregnancy: prevalence and correlates in two Cape Town peri-urban settlements. Reprod Health. 2011;8:9.

117. Rwakarema M, Premji SS, Nyanza EC, Riziki P, Palacios-Derflingher L. Antenatal depression is associated with pregnancy-related anxiety, partner relations, and wealth in women in Northern Tanzania: a crosssectional study. BMC Womens Health. 2015;15:68.

118. Heyningen TV, Myer L, Onah M, Tomlinson M, Field S, Honikman S. Antenatal depression and adversity in urban South Africa. J Affect Disord. 2016:203:121-9.

119. Pinheiro RT, da Cunha Coelho FM, da Silva RA, de Avila QL, de Mattos Souza LD, Castelli RD, de Matos MB, Pinheiro KA. Suicidal behavior in pregnant teenagers in southern Brazil: social, obstetric and psychiatric correlates. J Affect Disord. 2012;136:520-5.

120. Supraja T, Thennarasu K, Satyanarayana VA, Seena T, Desai G, Jangam KV Chandra PS. Suicidality in early pregnancy among antepartum mothers in urban India. Arch Womens Ment Health. 2016;19:1101-8.

121. Salameh T. Factors influencing postpartum depression among Jordanian women. Amman, Jordan: University of Jordan, Faculty of Nursing; 2006.

122. Hatton D, Harrison-Hohner J, Matarazzo J, Edwards P, Lewy A, Davis L. Missed antenatal depression among high risk women: a secondary analysis. Arch Womens Ment Health. 2007;10:121-3.

123. Ismail R. Analisis item, uji reliabilitas dan validitas kuesioner dukungan sosial (KDS) untuk ibu hamil dan nifas. Jakarta: Universitas Indonesia; 2001.

124. Dalgard OS, Dowrick C, Lehtinen V, Vazquez-Barquero JL, Casey P, Wilkinson G, Ayuso-Mateos JL, Page H, Dunn G, Group O. Negative life events, social support and gender difference in depression. Soc Psychiatry Psychiatr Epidemiol. 2006;41:444-51.

125. Stansfeld S, Marmot M. Deriving a survey measure of social support: the reliability and validity of the Close Persons Questionnaire. Soc Sci Med. 1992;35:1027-35.

126. Turner RJ, Grindstaff CF, Phillips N. Social support and outcome in teenage pregnancy. J Health Soc Behav. 1990;31:43-57.

127. Curry MA, Campbell RA, Christian M. Validity and reliability testing of the prenatal psychosocial profile. Res Nurs Health. 1994;17:127-35.

128. Chou K. Assessing Chinese adolescents'social support: The multidimensionalscale of perceived social support. Pers Individ Dif. 2000;28:299-307.

129. Pascoe J, lalongo NS, Horn W, Reinhart M, Perradatto D. The reliability and validity of the maternal social support index. Fam Med. 1988:20:271-6.

130. Kendler KS, Myers J, Prescott CA. Sex differences in the relationship between social support and risk for major depression: a longitudinal study of opposite-sex twin pairs. Am J Psychiatry. 2005;162:250-6.

131. Xiao $\mathrm{S}$. The theoretical basis and research application of social support rating scale. J Clin Psychiatry. 1994;4:98-100.

132. Rizwan M, Syed N. Urdu translation and psychometric properties of social provision scale. Int J Educ Psychol Assess. 2010;4.

133. Fydrich T, Sommer G, Brähler E. Fragebogen zur Sozialen Unterstützung: F-SozU; Manual. Hogrefe; 2007

134. Kafetsios K. Social support questionnaire-short form. The psychometric instruments in Greece Ellinika Grammata, Athens. 2002:373

135. Fydrich T, Geyer M, Hessel A, Sommer G, Brähler E. Fragebogen zur sozialen Unterstützung (F-SozU): Normierung an einer repräsentativen Stichprobe. Diagnostica. 1999;45:212-6.

136. Cohen S, Mermelstein R, KamarckT, Hoberman HM. Measuring the functional components of social support. In: Social support: theory, research and applications. Dordrecht: Springer; 1985. p. 73-94.

137. Weinert C, Brandt PA. Measuring social support with the Personal Resource Questionnaire. West J Nurs Res. 1987;9:589-602. 
138. Turner RJ, Marino F. Social support and social structure: a descriptive epidemiology. J Health Soc Behav. 1994;35:193-212.

139. Thoits PA. Social support as coping assistance. J Consult Clin Psychol. $1986 ; 54: 416$.

140. Alden LE, Mellings TM. Generalized social phobia and social judgments: the salience of self-and partner-information. J Anxiety Disord. 2004;18:143-57.

141. Paykel ES. Life events, social support and depression. Acta Psychiatr Scand Suppl. 1994;377:50-8.

142. Michalak EE, Wilkinson C, Hood K, Dowrick C, Wilkinson G. Seasonality, negative life events and social support in a community sample. $\mathrm{Br} J$ Psychiatry. 2003;182:434-8.

143. Chojenta C, Loxton D, Lucke J. How do previous mental health, social support, and stressful life events contribute to postnatal depression in a representative sample of Australian women? J Midwifery Womens Health. 2012;57:145-50.

144. Khazaeian S, Kariman N, Ebadi A, Nasiri M. The impact of social capital and social support on the health of female-headed households: a systematic review. Electron Phys. 2017;9:6027.

145. Stuart-Parrigon K, Stuart S. Perinatal depression: an update and overview. Curr Psychiatry Rep. 2014;16:468.

146. Ghasemipoor M, Jahanbakhsh GS. The relationship between the social support and mental health in Lorestan university students in 2009. YAFTEH. 2010;1 (2):57-64.

147. Kamran F. Ershadikh. Discovering social capital and mental health relationship. Soc Res (Islamic Azad University Roudehen Branch). 2009:2(3):29-54
148. Bullock L, Wells J, Duff G, Hornblow A. Telephone support for pregnant women: outcome in late pregnancy. N Z Med J. 1995;108:476-8.

149. Snaith VJ, Hewison J, Steen IN, Robson SC. Antenatal telephone support intervention with and without uterine artery Doppler screening for low risk nulliparous women: a randomised controlled trial. BMC Pregnancy Childbirth. 2014;14:1-11.

150. Côté-Arsenault D, Schwartz K, Krowchuk H, McCoy TP. Evidence-based intervention with women pregnant after perinatal loss. MCN Am J Matern Child Nurs. 2014;39:177-86.

151. UN Secretary General's high level panel on women's economic empowerment, leave no one behind: a call to action for gender equality and women's economic empowerment. https://www.empowerwomen. org/-/media/files/un\%20women/empowerwomen/resources/hlp\% 20briefs/unhlp\%20full\%20report.pdf?la=en.

152. Bourdieu P. The fine distinctions. Frankfurt am Main: Suhrkamp; 1982. (in German).

153. Jenkins R, Njenga F, Okonji M, Kigamwa P, Baraza M, Ayuyo J, Singleton N, McManus S, Kiima D. Prevalence of common mental disorders in a rural district of Kenya, and socio-demographic risk factors. Int J Environ Res Public Health. 2012;9:1810-9.

\section{Publisher's Note}

Springer Nature remains neutral with regard to jurisdictional claims in published maps and institutional affiliations.
Ready to submit your research? Choose BMC and benefit from:

- fast, convenient online submission

- thorough peer review by experienced researchers in your field

- rapid publication on acceptance

- support for research data, including large and complex data types

- gold Open Access which fosters wider collaboration and increased citations

- maximum visibility for your research: over $100 \mathrm{M}$ website views per year

At BMC, research is always in progress.

Learn more biomedcentral.com/submissions 